\title{
Finite strain analysis of size effects in wedge indentation into a Face-Centered Cubic
} (FCC) single crystal

Lynggaard, J.; Nielsen, K. L.; Niordson, C. F.

\section{Published in:}

European Journal of Mechanics A - Solids

Link to article, DOI:

10.1016/j.euromechsol.2019.02.008

Publication date:

2019

Document Version

Peer reviewed version

Link back to DTU Orbit

Citation (APA):

Lynggaard, J., Nielsen, K. L., \& Niordson, C. F. (2019). Finite strain analysis of size effects in wedge indentation into a Face-Centered Cubic (FCC) single crystal. European Journal of Mechanics A - Solids, 76, 193-207. https://doi.org/10.1016/j.euromechsol.2019.02.008

\section{General rights}

Copyright and moral rights for the publications made accessible in the public portal are retained by the authors and/or other copyright owners and it is a condition of accessing publications that users recognise and abide by the legal requirements associated with these rights.

- Users may download and print one copy of any publication from the public portal for the purpose of private study or research.

- You may not further distribute the material or use it for any profit-making activity or commercial gain

- You may freely distribute the URL identifying the publication in the public portal 


\section{Accepted Manuscript}

Finite strain analysis of size effects in wedge indentation into a Face-Centered Cubic (FCC) single crystal

J. Lynggaard, K.L. Nielsen, C.F. Niordson

PII: S0997-7538(18)30633-8

DOI: $\quad$ https://doi.org/10.1016/j.euromechsol.2019.02.008

Reference: EJMSOL 3742

To appear in: European Journal of Mechanics / A Solids

Received Date: 28 August 2018

Revised Date: 6 February 2019

Accepted Date: 11 February 2019

Please cite this article as: Lynggaard, J., Nielsen, K.L., Niordson, C.F., Finite strain analysis of size effects in wedge indentation into a Face-Centered Cubic (FCC) single crystal, European Journal of Mechanics / A Solids (2019), doi: https://doi.org/10.1016/j.euromechsol.2019.02.008.

This is a PDF file of an unedited manuscript that has been accepted for publication. As a service to our customers we are providing this early version of the manuscript. The manuscript will undergo copyediting, typesetting, and review of the resulting proof before it is published in its final form. Please note that during the production process errors may be discovered which could affect the content, and all legal disclaimers that apply to the journal pertain. 


\title{
Finite strain analysis of size effects in wedge indentation into a Face-Centered Cubic (FCC) single crystal
}

\author{
J. Lynggaard ${ }^{\mathrm{a}, *}$, K.L. Nielsen ${ }^{\mathrm{a}}$, C.F. Niordson ${ }^{\mathrm{a}}$ \\ ${ }^{a}$ Department of Mechanical Engineering, Section of Solid Mechanics, Technical University \\ of Denmark, DK-2800 Kongens Lyngby, Denmark
}

\begin{abstract}
The size effect in wedge indentation of an FCC single crystal is investigated. Conventional plasticity fails to describe the mechanical response of crystalline materials at the micron level due to the accumulation of Geometric-Necessary Dislocations (GNDs) which experiments have shown to introduce a size-dependent increase in the apparent yield stress and subsequent hardening. GND-densities scale with the gradient of plastic deformation and their effect on the mechanical response is here modelled by adopting a dissipative strain gradient single-crystal plasticity theory. Numerical solutions to the classical wedge indentation problem are obtained from a purpose-built Finite Element model accounting for finite strains. A special 2D plane strain set-up, with three effective in-plane slip systems, is adopted to comply with state-of-the-art experimental results. The indentation process is modelled for a nearly flat wedge as well as a wedge with an included angle of $90^{\circ}$. The distribution of slip and the GND-densities are investigated and compared to conventional plasticity predictions.
\end{abstract}

Keywords:

Crystal plasticity, Strain gradient plasticity, Finite elements, Indentation, Finite strain

\section{Introduction}

The growing interest in size effects on material behaviour is partly a result of developments within material science, where micron-scale coatings now play a major role in the manufacturing industry. This has led to a request for more accurate predictions of material behaviour on such small scales. Since the early 1990s, several experimental investigations on metals have confirmed the general trend that 'smaller is stronger'; a size-dependence that conventional plasticity fails to capture. Indentation experiments show a significant increase

\footnotetext{
* Corresponding author

Email addresses: jullyn@mek.dtu.dk (J. Lynggaard), KIN@mek.dtu.dk (K.L. Nielsen), CN@mek.dtu.dk (C.F. Niordson)
} 
in the measured material hardness on the micron scale (Stelmashenko et al. (1993) and Ma and Clarke (1995)). The mechanism behind this size effect is the generation of so-called Geometrically Necessary Dislocations (GNDs) that accommodate lattice curvature (Ashby (1970)), and thus accumulate in areas with large strain gradients. An early formulation of isotropic Strain Gradient Plasticity (SGP), using a minimum number of additional constitutive variables of dimension length, was proposed by Fleck et al. (1994) to account for size effects, and the theory was further developed in what is now a vast body of research that includes Fleck and Hutchinson (1997, 2001), Gudmundson (2004) and Fleck and Willis (2009). Although most of the literature focus on so-called higher order theories, employing higher order boundary conditions associated with plastic flow (Gao et al. (1999), Evers et al. (2004),Kuroda and Tvergaard (2006)), theories that retain the structure of a conventional boundary value problem have also been proposed (see e.g. Acharya and Bassani (2000) and Huang et al. (2000)). A corresponding research track in higher order Strain Gradient Crystal Plasticity (SGCP) theory includes Gurtin (2002), Bardella (2006a,b), Gurtin et al. (2007), Borg (2007) among others.

The finite strain gradient crystal plasticity theory here developed is a generalization of the small strain framework of Niordson and Kysar (2013), which is based on the mathematical foundation laid out for isotropic theory by Fleck and Willis (2009). The finite strain formulation is described within an updated Lagrangian framework and inspired by the finite strain generalization of the theory by Fleck and Hutchinson (2001) developed in Niordson and Redanz (2004).

The objective of the present study is to model size-effects in micron scale wedge indentation into an FCC single crystal, in terms of; i) hardness, and ii) slip and GND fields. Analyses are carried out for two wedge geometries; a nearly flat indenter with an included wedge angle of $170^{\circ}$ and an indenter with an included angle of $90^{\circ}$. The problem is investigated within a $2 \mathrm{D}$ plane strain setting that fit a specific class of orientations for FCC single crystals. Comparisons are made to solutions from the literature including Zhang et al. (2014).

The present paper is structured as follows: The material model is outlined in Section 2, the numerical solution method in Section 3, the model setup including the effective slip systems and the wedge indentation problem are defined in Section 4, results are presented and discussed in Section 5 leading to the concluding remarks in Section 6.

\section{Material model}

The material model employed is analogous to that of Niordson and Kysar (2013), but here including only dissipative gradient effects. The model is developed in detail in the finite strain context employing an updated Lagrangian approach in a Cartesian coordinate system.

Plastic deformation is assumed to occur due to slip, $\dot{\gamma}^{(\alpha)}$, on a number of slip systems $(\alpha)$, with current slip plane normals $m_{i}^{(\alpha)}$ and slip directions $s_{i}^{(\alpha)}$. Slip 
plane normals and slip directions in the underformed configuration are denoted $m_{i 0}^{(\alpha)}$ and $s_{i 0}^{(\alpha)}$, respectively. The plastic strain rate tensor is given by

$$
\dot{\varepsilon}_{i j}^{p}=\sum_{(\alpha)} \dot{\gamma}^{(\alpha)} \mu_{i j}^{(\alpha)}
$$

with the Schmid orientation defined by

$$
\mu_{i j}^{(\alpha)}=\frac{1}{2}\left(s_{i}^{(\alpha)} m_{j}^{(\alpha)}+s_{j}^{(\alpha)} m_{i}^{(\alpha)}\right)
$$

The slip rate on an individual slip system $(\alpha)$ is assumed to follow the power-law relation

$$
\tau_{e}^{(\alpha)}=\tau_{0}^{(\alpha)}\left(\frac{\dot{\gamma}_{e}^{(\alpha)}}{\dot{\gamma}_{0}^{(\alpha)}}\right)^{m}
$$

where $\tau_{e}^{(\alpha)}$ is an effective resolved shear stress, $\tau_{0}^{(\alpha)}$ is the initial slip resistance, $\dot{\gamma}_{0}^{(\alpha)}$ is the reference slip rate, $\dot{\gamma}_{e}^{(\alpha)}$ is the effective plastic slip rate, and $m$ is the slip rate hardening exponent. The material is here assumed to be elasticperfectly plastic, implying that the slip resistance $\tau_{0}^{(\alpha)}$ is constant and does not develop with slip.

The principle of virtual work for the strain gradient crystal plasticity theory can be written as (see Gurtin (2002); Niordson and Kysar (2013))

$$
\begin{array}{r}
\int_{V}\left(\sigma_{i j} \delta \dot{\varepsilon}_{i j}+\sum_{\alpha}\left(q^{(\alpha)}-\tau^{(\alpha)}\right) \delta \dot{\gamma}^{(\alpha)}+\sum_{(\alpha)} \xi^{(\alpha)} s_{i}^{(\alpha)} \delta \dot{\gamma}_{, i}^{(\alpha)}\right) \mathrm{d} V \\
=\int_{S}\left(T_{i} \delta \dot{u}_{i}+\sum_{\alpha} r^{(\alpha)} \delta \dot{\gamma}^{(\alpha)}\right) \mathrm{d} S
\end{array}
$$

where, $V$ and $S$ here denote the current volume and surface of the body, respectively. The Cauchy stress $\sigma_{i j}$ is work conjugate to the total strain rate, $\dot{\varepsilon}_{i j}$, which is taken as the sum of the elastic strain rate, $\dot{\varepsilon}_{i j}^{e}$, and the plastic strain rate, $\dot{\varepsilon}_{i j}^{p}$. The Schmid stress is given by; $\tau^{(\alpha)}=\sigma_{i j} \mu_{i j}^{(\alpha)}$. The micro-stress, $q^{(\alpha)}$, is work conjugate to the slip rate, $\dot{\gamma}^{(\alpha)}$, and the higher order stress, $\xi^{(\alpha)}$, is work conjugate to the slip rate gradient resolved onto the slip direction $s_{i}^{(\alpha)} \dot{\gamma}_{, i}^{(\alpha)}$. On the right hand side of Eq. (4), $T_{i}$ is the conventional traction vector work conjugate to the displacement rate, $\dot{u}_{i}$, and $r^{(\alpha)}$ denote the higher order tractions that are work conjugate to the slip rates, $\dot{\gamma}^{(\alpha)}$.

The strong form of the equilibrium equations (in absence of body forces), consistent with the above principle of virtual work, are given by

$$
\begin{gathered}
\sigma_{i j, j}=0 \\
q^{(\alpha)}-\tau^{(\alpha)}-\xi_{, i} s_{i}^{(\alpha)}=0
\end{gathered}
$$


in the volume $V$ and

$$
\begin{gathered}
T_{i}=\sigma_{i j} n_{j} \\
r^{(\alpha)}=\xi^{(\alpha)} s_{i}^{(\alpha)} n_{i}
\end{gathered}
$$

on the surface $S$.

Following Fleck and Hutchinson (2001) and Gudmundson (2004) for isotropic plasticity and Bardella (2006a) and Borg (2007) for crystal plasticity, an effective slip quantity is defined incrementally from the slip rate and the slip rate gradient according to

$$
\left(\dot{\gamma}_{e}^{(\alpha)}\right)^{2}=\left(\dot{\gamma}^{(\alpha)}\right)^{2}+\left(L_{D}^{(\alpha)} \dot{\gamma}_{, i}^{(\alpha)} s_{i}^{(\alpha)}\right)^{2}
$$

where $L_{D}$ denotes a so-called dissipative length scale. A visco-plastic potential for each of the slip systems is defined as

$$
\Phi^{(\alpha)}\left[\dot{\gamma}_{e}^{(\alpha)}\right]=\int_{0}^{\dot{\gamma}_{e}^{(\alpha)}} \tau_{e}^{(\alpha)}\left[\dot{\gamma}_{e}^{(\alpha) \prime}\right] \mathrm{d} \dot{\gamma}_{e}^{(\alpha) \prime}
$$

The potential enables the identification of the dissipative stress quantities upon investigation of the variation of the potential $\partial \Phi=\tau_{e}^{(\alpha)} \partial \dot{\gamma}_{e}^{(\alpha)}$ through

$$
\partial \Phi=q^{(\alpha)} \partial \dot{\gamma}^{(\alpha)}+\xi^{(\alpha)} s_{i}^{(\alpha)} \partial \dot{\gamma}_{, i}^{(\alpha)}
$$

showing that the micro-stress, $q^{(\alpha)}$, and the higher-order stress, $\xi^{(\alpha)}$, are given by

$$
q^{(\alpha)}=\frac{\tau_{e}^{(\alpha)}}{\dot{\gamma}_{e}^{(\alpha)}} \dot{\gamma}^{(\alpha)} \quad \text { and } \quad \xi^{(\alpha)}=\frac{\tau_{e}^{(\alpha)}}{\dot{\gamma}_{e}^{(\alpha)}}\left(L_{D}^{(\alpha)}\right)^{2} \dot{\gamma}_{, i}^{(\alpha)} s_{i}^{(\alpha)}
$$

By substituting Eq. (12) into Eq. (9), the following expression is obtained for the effective stress:

$$
\left(\tau_{e}^{(\alpha)}\right)^{2}=\left(q^{(\alpha)}\right)^{2}+\frac{1}{L_{D}^{2}}\left(\xi^{(\alpha)}\right)^{2}
$$

\section{Solution method and the numerical framework}

To solve for the slip rate fields, $\dot{\gamma}^{(\alpha)}$, Minimum Principle I of Fleck and Willis (2009) is used as formulated by Niordson and Kysar (2013) for crystal plasticity. Higher order equilibrium is satisfied by the minimizing field among all kinematically admissible (non-zero) fields, $\dot{\gamma}^{(\alpha) *}$, for the functional

$$
H^{(\alpha)}=\inf _{\dot{\gamma}^{(\alpha) *}} \int_{V}\left(\Phi\left[\dot{\gamma}_{e}^{(\alpha) *}\right]-s_{i j} \mu_{i j}^{(\alpha)} \dot{\gamma}^{(\alpha) *}\right) \mathrm{d} V-\int_{S_{T}} r^{(\alpha)} \dot{\gamma}^{(\alpha) *} \mathrm{~d} S .
$$

Here, $s_{i j}=\sigma_{i j}-\delta_{i j} \sigma_{k k}$ denotes the deviatoric part of the Cauchy stresses. Stationarity of the functional $H^{(\alpha)}$ results in the variational statement

$$
\int_{V}\left(q^{(\alpha)} \delta \dot{\gamma}^{(\alpha)}+\xi^{(\alpha)} s_{i} \delta \dot{\gamma}_{, i}^{(\alpha)}\right) \mathrm{d} V=\int_{V}\left(s_{i j} \mu_{i j}^{(\alpha)} \dot{\gamma}^{(\alpha)}\right) \mathrm{d} V+\int_{S} r^{(\alpha)} \dot{\gamma}^{(\alpha)} \mathrm{d} S
$$


Note here that all terms related to energetic contributions are left out in this study and further work is needed on the finite strain formulation to account for such effects.

A forward Euler time-stepping algorithm is employed, where for each time increment the slip rate fields are obtained iteratively based on the discretized versions of Eq. (15) for each slip system as laid out in Niordson and Kysar (2013). For each time increment, the slip rate fields are determined based on the stress state at the start of the increment, $s_{i j}$, and the current crystallographic bases vectors, which are updated according to

$$
\begin{aligned}
& s_{i}^{*}=F_{i j}^{e} s_{j} \\
& m_{i}^{*}=m_{j} F_{j i}^{e-1}
\end{aligned}
$$

with $F_{i j}^{e}$ and $F_{i j}^{e-1}$ denoting the elastic deformation gradient and its inverse, respectively (see e.g. Peirce et al. (1983)). A dicretized finite element solution for the slip rate field is obtained based on four node quadrilateral elements with bilinear shape functions. The slip rate and its gradients are interpolated by

$$
\dot{\gamma}^{(\alpha)}=\sum_{N=1}^{12} M^{N} \dot{\gamma}_{N}^{(\alpha)} \quad \text { and } \quad \dot{\gamma}_{, i}^{(\alpha)}=\sum_{N=1}^{12} M_{, i}^{N} \dot{\gamma}_{N}^{(\alpha)}
$$

for each slip system $(\alpha)$, with $M^{N}$ being a matrix containing the shape functions. Further details on the solution method can be found in Niordson and Kysar (2013) for the corresponding small strain problem. In the present finite strain context it is of key essence that the slip rate fields are solved in the deformed configuration based on the stress deviator field before the next displacement increment, along with boundary conditions for either the slip rate field, $\dot{\gamma}^{(\alpha)}$, or the surface work conjugate, $r^{(\alpha)}$.

Having solved for the slip rate fields, the nodal displacement increments are subsequently determined based on the Pinciple of Virtual Work, specialized for $\delta \dot{\gamma}^{(\alpha)}=0$ :

$$
\int_{V} \sigma_{i j} \delta \dot{\varepsilon}_{i j} \mathrm{~d} V=\int_{S} T_{i} \delta \dot{u}_{i} \mathrm{~d} S
$$

Following McMeeking and Rice (1975), the conventional Principle of Virtual Work is rewritten in a reference frame (see also Niordson and Redanz (2004))

$$
\int_{V_{0}} P_{i j} \delta \dot{u}_{j, i} \mathrm{~d} V=\int_{S_{0}} T_{i}^{0} \delta \dot{u}_{i} \mathrm{~d} S
$$

where $P_{i j}$ is the first Piola-Kirchhoff stress and $T_{i}^{0}$ is the nominal traction vector. The incremental form simply reads

$$
\int_{V_{0}} \dot{P}_{i j} \delta \dot{u}_{j, i} \mathrm{~d} V=\int_{S_{0}} \dot{T}_{i}^{0} \delta \dot{u}_{i} \mathrm{~d} S
$$

With the goal of formulating the elastic constitutive relation in terms of the Jaumann rate of the Kirchhoff stress, $\varsigma_{i j}$, the following identity for the increment 
of the first Piola-Kirchhoff stress is employed (see Hill (1959) and McMeeking and Rice (1975))

$$
\dot{P}_{i j}=\varsigma_{i j}-\sigma_{k j} \dot{\varepsilon}_{i k}-\sigma_{i k} \dot{\varepsilon}_{j k}+\sigma_{i k} \dot{u}_{j, k}
$$

to express the Principle of Virtual Work as

$$
\int_{V_{0}}\left(\varsigma_{i j} \delta \dot{\varepsilon}_{i j}-\sigma_{i j}\left(2 \dot{\varepsilon}_{i k} \delta \dot{\varepsilon}_{k j}-\dot{u}_{k, j} \delta \dot{u}_{k, i}\right)\right) \mathrm{d} V=\int_{S_{0}} \dot{T}_{i}^{0} \delta \dot{u}_{i} \mathrm{~d} S
$$

as obtained by McMeeking and Rice (1975).

Employing the hypo-elastic constitutive relation $\varsigma_{i j}=L_{i j k l}\left(\dot{\varepsilon}_{k l}-\dot{\varepsilon}_{k l}^{p}\right)$, where $L_{i j k l}$ is the isotropic elastic stiffness tensor, leads to

$\int_{V_{0}}\left(L_{i j k l} \dot{\varepsilon}_{k l} \delta \dot{\varepsilon}_{i j}-\sigma_{i j}\left(2 \dot{\varepsilon}_{i k} \delta \dot{\varepsilon}_{k j}-\dot{u}_{k, j} \delta \dot{u}_{k, i}\right)\right) \mathrm{d} V=\int_{S_{0}}\left(L_{i j k l} \dot{\varepsilon}_{k l}^{p} \delta \dot{\varepsilon}_{i j}+\dot{T}_{i}^{0} \delta \dot{u}_{i}\right) \mathrm{d} S$

which serves as a basis for a finite element dicretization where eight node quadrilateral elements are used to interpolate displacements. Note that the right hand side of Eq. (22) includes the viscoplastic contributions that are known before each time increment from the solution of Minimum Principle I of Fleck and Willis (2009).

Displacement rates and strain rates are interpolated according to

$$
\delta \dot{u}_{i}=\sum_{n=1}^{16} N_{i}^{n} \delta \dot{D}^{n}, \quad \delta \dot{\varepsilon}_{i j}=\sum_{n=1}^{16} E_{i j}^{n} \delta \dot{D}^{n}
$$

Where $N_{i}^{n}$ contains the nodal shape functions and $E_{i j}^{n}=\frac{1}{2}\left(N_{i, j}^{n}+N_{j, i}^{n}\right)$. This leads to the following discretized version of Eq. (22) for a single element

$$
\begin{array}{r}
\sum_{N=1}^{16} \sum_{M=1}^{16} \int_{V_{e}}\left(L_{i j k l} E_{i j}^{M} E_{k l}^{N}-\sigma_{i j}\left(2 E_{i k}^{M} E_{k j}^{N}-N_{k, j}^{M} N_{k . i}^{N}\right)\right) \mathrm{d} V \cdot \dot{D}^{M}= \\
\sum_{N=1}^{16} \int_{S_{e}}\left(L_{i j k l} \dot{\varepsilon}_{i j}^{p} E_{i j}^{N}+\dot{T}_{i}^{0} N_{i}^{N}\right) \mathrm{d} S
\end{array}
$$

\section{Problem formulation}

The wedge indentation problem investigated is illustrated in Figure 1. The material domain consists of a single crystal material, with a Face Cubic Centered (FCC) crystal structure. Elastic deformations are approximated as isotropic. For specific crystal orientations, a state of plane strain prevails well inside the crystal and thus, a 2D model set-up is adopted. The geometric requirement is that no out-of-plane plastic deformations occur during the indentation as will be discussed in Section 4.1. The wedge is assumed rigid and full friction is assumed such that a material point which comes into the contact will follow the path of the wedge. The angle between the wedge and the undeformed indenter 
surface is denoted $\phi$ and the wedge is modelled with an initial flat tip area, $a_{c}^{0}$, to regularize the numerical solution upon initial contact. The level of indentation is quantified by the nominal indentation distance, $\delta$. As the indentation progresses, the contact length between the wedge and the material domain will increase and the total contact length in the deformed configuration is denoted $a_{c}$. Figure 1 illustrates the contact length in a situation where the material experiences "sinkin", but the case of "pile-up" is captured appropriately by the model as well (see discussion on "sink-in" and "pile-up" in Section 5). A semi-circular region of radius $r_{0}$ is modelled sufficiently large to suppress boundary effects. The wedge is pressed into the domain at an increasing rate such that the indentation rate normalized by the current contact is constant, $\dot{\varepsilon}_{v}=\dot{\delta} / a_{c}=$ constant. The macroscopic hardness of the domain material is defined as the applied force, measured by the total reaction force on the wedge, divided by the projected contact area in the deformed configuration, $H=F / a_{c} t$, where $\mathrm{t}$ is the thickness of the material domain considered.

The densities of Geometrically Necessary Dislocations (GNDs) are evaluated in the deformed configuration according to

$$
\rho_{G N D}^{(\alpha)}=-\frac{1}{b} \gamma_{, i}^{(\alpha)} s_{i}^{(\alpha)},
$$

where $b$ is the Burgers vector. In the results presented, this density is normalized by a reference density defined as

$$
\rho_{0}^{(\alpha)}=\frac{\gamma_{0}^{(\alpha)}}{b 2 a_{c}} .
$$

The input parameters used in this study are listed in Table 1. The material parameters were chosen to represent single crystal aluminum which is known to have an FCC crystal structure that is nearly elastically isotropic.

The employed Finite Element mesh is depicted in Figure 2 and is constructed to have the highest resolution closest to the indenter. The initial contact length covers the four top elements closest to $\left(x_{1}, x_{2}\right)=(0,0)$. The mesh size was chosen to minimize the numerical errors while maintaining a reasonable computational time. In the numerical model, the contact length in the deformed configuration is only updated when a new node in the Finite Element mesh comes into contact with the wedge. This discrete update of the contact length will lead to vertical drops in the hardening curves that scale with mesh size (as seen in the results).

\subsection{Single crystal effective slip systems}

A planar Face Centred Cubic (FCC) single crystal oriented along the (110) plane subject to a line loading along the $<110>$ direction is considered. Following Rice (1987), three pairs of slip systems can be identified as three effective edge slip systems that give rise to plane strain deformation for such conditions (see Figure 1). This setting has been investigated in a number of theoretical and experimental studies including Rice (1987), Kysar et al. (2007), Niordson 
and Kysar (2013), Kysar and Gan (2016), Juul et al. (2018b) and Lewandowski and Stupkiewicz (2018).

By denoting the effective slip systems $(\alpha)$ and the paired crystallographic slip systems as $(\alpha a)$ and $(\alpha b)$, all effective slip parameters can be calculated with respect to the crystallographic slip parameters according to Table 2 . The assumption of simultaneous slip implies that; slip in one of the effective slip systems $(\alpha)$ occurs as a consequence of simultaneous slip in the correspondingly paired crystallographic slip systems $(\alpha a)$ and $(\alpha b)$.

In Table 2 , the $\beta^{(\alpha)}$ factor defines the ratio between the slip on the crystallographic slip systems and the effective slip systems, $s_{i}^{(\alpha)} m_{j}^{(\alpha)}$ is the effective deformation tensor and $s_{i}^{(\alpha a)} m_{j}^{(\alpha a)}+s_{i}^{(\alpha b)} m_{j}^{(\alpha b)}$ is the sum of the pairwise crystallographic deformation tensors. The ratio $\beta^{(\alpha)}$ is independent whether it is calculated based on the reference or the current crystallographic basis vectors. From this, the reference slip rate for the effective slip systems can be calculated from the crystallographic reference slip rate $\dot{\gamma}_{0}$ as

$$
\dot{\gamma}_{0}^{(\alpha)}=\beta^{(\alpha)} \dot{\gamma}_{0}
$$

Let the ratio between the resolved shear stress in the effective slip systems and crystallographic slip systems be defined as

$$
\lambda^{(\alpha)}=\frac{\tau^{(\alpha)}}{\tau^{(\alpha a)}}=\frac{\tau^{(\alpha)}}{\tau^{(\alpha b)}} .
$$

This implies that the initial slip resistance in the effective slip systems can be calculated from the crystallographic input value $\tau_{0}$ as; $\tau_{0}^{(\alpha)}=\lambda^{(\alpha)} \tau_{0}$. The following relation between the two ratios holds (see Niordson and Kysar (2013)):

$$
\beta^{(\alpha)} \lambda^{(\alpha)}=2 .
$$

To find an expression for the ratio between the crystallographic dissipative length scale, $L_{D}$, and the effective dissipative length scale, $L_{D}^{(\alpha)}$, the crystallographic effective slip rate is considered. Using the same quadratic definition for both the effective slip and the crystallographic slip system $(\alpha a)$, as in Eq. (9), yields:

$$
\left(\dot{\gamma}_{e}^{(\alpha a)}\right)^{2}=\left(\dot{\gamma}^{(\alpha a)}\right)^{2}+\left(L_{D} \dot{\gamma}_{, i}^{(\alpha a)} s_{i}^{(\alpha a)}\right)^{2}
$$

Multiplying through with $\left(\beta^{(\alpha)}\right)^{2}$ and using $\beta^{(\alpha)} \dot{\gamma}_{e}^{(\alpha a)}=\dot{\gamma}_{e}^{(\alpha)}, \beta^{(\alpha)} \dot{\gamma}^{(\alpha a)}=\dot{\gamma}^{(\alpha)}$, and $\beta^{(\alpha)} \dot{\gamma}_{, i}^{(\alpha a)}=\dot{\gamma}_{, i}^{(\alpha)}$, the following expression for the effective slip rate must hold

$$
\left(\dot{\gamma}_{e}^{(\alpha)}\right)^{2}=\left(\dot{\gamma}^{(\alpha)}\right)^{2}+\left(L_{D} \dot{\gamma}_{, i}^{(\alpha)} s_{i}^{(\alpha a)}\right)^{2} .
$$

Comparing Eqs. (9) and (31) one finds

$$
\dot{\gamma}_{, i}^{(\alpha)} s_{i}^{(\alpha a)}=\mu^{(\alpha)} \dot{\gamma}_{, i}^{(\alpha)} s_{i}^{(\alpha)}
$$


Where the following definition $L_{D}^{(\alpha)} / L_{D}=\mu^{(\alpha)}$ is used. Due to plane strain conditions, $\dot{\gamma}_{3}^{(\alpha)}=0$, and it may be shown that

$$
\mu^{(\alpha)}=\frac{L_{D}^{(\alpha)}}{L_{D}}=s_{i}^{(\alpha a)} s_{i}^{(\alpha)}=s_{i}^{(\alpha b)} s_{i}^{(\alpha)}
$$

where the last expression results from symmetry considerations. ${ }^{1}$

\section{Results}

All results are expressed in dimensionless form. Lengths are normalized with respect to the initial contact length, $a_{c}^{0}$, which in an experimental setup may be interpreted as a measure of the tip radius of an imperfect wedge indenter. For comparison, the results for conventional crystal plasticity, with $L_{D}=0$, are also shown. Two wedge angles are considered; a nearly flat wedge with $\phi=5^{\circ}$ (included angle of $170^{\circ}$ ) and a wedge with $\phi=45^{\circ}$ (included angle of $90^{\circ}$ ).

\subsection{Mesh convergence}

Mesh convergence is analyzed by considering the macroscopic hardness for seven different mesh resolutions (see Table 3). The macroscopic hardness as a function of indentation depth is displayed in Figure 3 for conventional plasticity $\left(L_{D}=0\right)$ and an included wedge angle of $170^{\circ}$. The hardness curves display distinct vertical drops (related to new nodal contacts), and the most reliable hardness values are to be found just after these drops. By considering only these points on the hardness curve, the hardness values seem to be nearly constant after a transient state of around $\delta / a_{c}^{0}=0.5$. For the more refined meshes, the predicted hardness level is well captured at lower indentation levels. This, however, comes at the cost of a higher overall computational time. When refining from Mesh No. 5 to Mesh No. 6 and 7, the gain in numerical accuracy is minor and therefore mesh no. 5 is used in the remaining analyses.

\subsection{Results using a nearly flat wedge indenter}

For conventional plasticity, the macroscopic hardness using a nearly flat indenter with an included angel of $170^{\circ}\left(\phi=5^{\circ}\right)$ is found to be $H / \tau_{0} \approx 6.83$.

The total slip for six different indentation depths is shown in Figure 4 here with the total slip calculated from the slip on the individual effective slip systems determined as

$$
\frac{\gamma^{T}}{\gamma_{0}}=\frac{\left|\gamma^{(1)}\right|}{\gamma_{0}^{(1)}}+\frac{\left|\gamma^{(2)}\right|}{\gamma_{0}^{(2)}}+\frac{\left|\gamma^{(3)}\right|}{\gamma_{0}^{(3)}},
$$

where

$$
\gamma_{0}^{(\alpha)}=\frac{\tau_{0}^{(\alpha)}}{G}
$$

\footnotetext{
${ }^{1}$ Note the exponent misprint in Niordson and Kysar (2013) in their equation equivalent to Eq. (33).
} 
Aside from an transient build-up phase (related to the flat part of the indenter tip, and the initial coarse discretization of the contact area), the response of the conventional plasticity model shows a field with a self-similar development, i.e. the shape of the field remains the same but increases in size in proportion to the current contact length. This effect is expected, since, besides from the initial contact length, there is only one independent dimensional parameter in the system (for a more detailed discussion on self-similar behaviour in relation to indentation see Juul et al. (2018b) and Juul et al. (2018a)). Once the field exhibiting self-similarity has evolved in the current numerical set-up, a constant hardness level with indentation depth is maintained as can be seen in Figure 3, Figure 6 and Figure 13 for conventional plasticity. The plastic slip and the GND-densities for the individual effective slip systems are shown in Figure 5 at a contact length of $a_{c} / a_{c}^{0}=15$. Here it may be seen, that the largest contributions to the total slip develops on slip systems (1) and (3), which are also seen to be mirrored versions of each other around the mirror plane at $x_{1}=0$. This is due to their mutual symmetry around this plane (see Figure 1), and thus the field plots for effective slip system (3) has been omitted in the remaining. Since the effective slip system (2) shares the symmetry plane $x_{1}=0$ with the indentation (see Figure 1), the slip and GND-density fields for effective slip system (2) are also symmetric around this plane. Another observation from Figure 5 is that most of the plastically deforming domain is dominated by one of the effective slip systems in different regions and large slip gradients can be seen near the slip region interfaces (also seen from Juul et al. (2018b)). The large slip gradients along these interfaces lead to narrow bands with high absolute values of GNDdensity, as seen in Figure 5 (e) and (f).

When introducing a length scale, i.e. going from conventional to strain gradient plasticity, the self-similar field evolution no longer exists, as the system now depends on a matetrial length parameter, which does not scale with indentation depth. This means that the solution, in terms of field shape and magnitude, now depends on the current contact length. The hardness curves for five different length scales are shown in Figure 6 together with the conventional plasticity curve. In the figure, a clear increase in hardness can be seen for the strain gradient solutions. For each length parameter the hardness curves converges towards the conventional curve, as the contact length increases relative to the material length parameter and the size effect becomes smaller. Another important observation is, that if the length parameter doubles, the curves converge half as fast as the smaller length parameter. This means that any hardness readings on a curve with length parameter $L_{D}$ at a contact length $a$, will be identical to a reading on a curve with length parameter $2 L_{D}$ at a contact length of $2 a$. When normalizing the first axis of the hardness curve with the length parameter, the hardness curves should therefore fall on top of each other. This is seen in Figure 7, where the curves predict the same indentation response after a transient period.

In the following field plots for a length parameter of $L_{D} / a_{c}^{0}=16$ is used. The first axis in these field plots are set to have a total range of $6 a_{c} / a_{c}^{0}$ and it is hereby dependent on the contact length in the deformed configuration for 
an easy comparison between different indentation levels. The total slip for four different contact lengths (indentation depths) are shown in Figure 8. It is seen that as the contact length increases, the shape of the total slip field becomes closer to the one predicted by conventional crystal plasticity (compare results to Figure 5). Note that all four plots differ significantly from the conventional plasticity predictions in locations where total slip gradients are large. This is even more clear when studying the individual slips and GND-densities for the effective slip systems. These are shown for $a_{c} / a_{c}^{0}=6$ in Figure 9 , for $a_{c} / a_{c}^{0}=12$ in Figure 10 and for $a_{c} / a_{c}^{0}=24$ in Figure 11. When comparing both the total slip and the individual slip for the plots including a length scale with the results from conventional plasticity in Figure 5, it is seen that the slip predictions for the gradient enhanced model are more smooth in appearance as gradients are penalized energetically. This also has the effect that significant regions of multislip exist in contrast to results for conventional plasticity (compare to Figure 5), and it predominantly observed for large ratios of material length scale to contact length, due to larger size effects. If only considering the slip activity on effective slip system (1) (Figure 9-11 (a)), the slip zones become more concentrated around the indentation tip and more detailed in appearance as the deformed contact length increases. Considering instead effective slip system (2) (Figure 9-11 (b)), the values for the gradient enhanced predictions were found to be far less than that of conventional crystal plasticity, with slip values far below one for contact lengths of $a_{c} / a_{c}^{0}=12$ and below. The narrow bands with high values of GND-density that were found for conventional plasticity are no longer observed in the gradient enhanced material (Figure 9-11 (c) and (d)). As a result of the smoother slip transitions, the GND-densities are markedly reduced, by a factor of more than ten, and the regions with highest GNDaccumulations are observed to be wider and less focused. When comparing the densities for effective slip system (1) at the different contact lengths, it is clear that the zones with positive values of the GND-density are almost non-existent for $a_{c} / a_{c}^{0}=6$, but they expand as the contact length increases. It is also worth to notice that the regions with the highest absolute GND-density moves towards the indentation tip as the contact length increases. Smaller GND-densities are linked to a harder macroscopic material response, so the observed GND-densities match the increase in macroscopic hardness that is apparent in Figure 6.

\subsection{Results using a wedge indenter of $90^{\circ}$ included angle}

Now considering the same input parameters, but changing the included wedge angle to $90^{\circ}\left(\phi=45^{\circ}\right)$, the total slip, individual slips, and GND-densities for conventional plasticity are shown in Figure 12. From these field plots it can be observed that the slip is much higher when compared to what was obtained with the nearly flat indenter - by a factor of about 20 . However, the slip distributions display essential similarities to the results obtained with the nearly flat indenter. The slip systems (1) and (3) are again the most dominant. The shape of the slip zone underneath the sharp indenter appears to be similar to that of the nearly flat indenter, but is greatly distorted in the $x_{2}$-direction due to the larger material deformations. This is also reflected in the slip gradients, 
for which the most notable differences are seen. The sharp lines with large slip gradients are not as well defined for the $90^{\circ}$ wedge, leading to GND-density plots that appears more smeared out, with the maximum GND-densities less than 10 times larger, and not about 20 times larger as the slip quantities.

The macroscopic hardness curves for different length scales are depicted in Figure 13. When compared to Figure 6, for the nearly flat indenter, it is clear that the hardness measurements are generally larger for the sharp indenter compared to the nearly flat indenter. For the conventional plasticity analysis, a hardness of $H / \tau_{0} \approx 8.34$ is found for the $90^{\circ}$ indenter (a $22 \%$ increase from the nearly flat indenter). Moreover, the increase in hardness, when applying a length scale, is even greater for the sharp indenter.

For the $90^{\circ}$ wedge, a pile-up effect may occur around the wedge, where material builds up above the original domain surface $x_{2}=0$ in the zones just around the wedge. This effect is attributed to the material strain hardening for elastic/plastic materials (also described in McElhaney et al. (1997)), and thus, gradient hardening affects it.

The total slip for four different contact lengths, when using a length scale of $L_{D} / a_{c}^{0}=16$, is shown in Figure 14. As it was also observed with the nearly flat indenter, it can be seen that the total slip converges towards the solution for conventional plasticity as the indentation contact length increases. Furthermore, it is observed that the pile-up effect that the conventional model exhibits is also seen when introducing the length scale effect when the contact length is $a_{c} / a_{c}^{0}=12$ and above. However, for $a_{c} / a_{c}^{0}=6$ and below the pile-up changes to sinking-in, where material not in contact with the wedge is deformed below the original surface. This effect can be directly related to the increase in gradient hardening on smaller scales. It was found that at a length scale of $L_{D} / a_{c}^{0}=2$ and $L_{D} / a_{c}^{0}=1$, as well as in the conventional plasticity analysis $\left(L_{D}=0\right)$, the pile-up effect started even before the first contact length update (first new nodal contact).

The slip and GND-densities for the individual effective slip systems are plotted in Figures $15-17$ for $a_{c} / a_{c}^{0}=6,12$, and 24 . Similar effects as for the nearly flat indenter can be observed. The change in slip becomes smoother by introducing the length scale effects which in turn results in lower and more smeared out GND-density fields. The zones with the highest accumulation again seem to be located further away from the indenter tip for smaller contact lengths. For the $90^{\circ}$ indenter, effective slip system (1) and (3) still gives the highest contributions to the total slip, but in contrast to results for the nearly flat indenter, effective slip system (2) has a notable contribution to the total slip even for the smallest indentation contact lengths.

\section{Conclusion}

Size-effects related to wedge indentation into an elastic-perfect plastic FCC single crystal material has been investigated, and the macroscopic hardness, the associated plastic slip, and the GND-densities are reported based on a newly developed strain gradient crystal plasticity framework accounting for finite strains. 
The numerical framework is based on the numerical small-strain framework of Niordson and Kysar (2013) in an updated Lagrangian formulation, with size effects introduced through a single dissipative length parameter, $L_{D}$. Three effective slip systems, which are $-57.4^{\circ}$ apart, are exploited to model the plane strain conditions of a specific crystal orientation. Special attention is on the evolution in plastic slip and GND distributions with increasing contact length for different length parameters, $L_{D}$, - ranging from $L_{D} / a_{c}^{0}=16$ to the conventional limit $L_{D}=0$ (no gradient effects). Furthermore, the development of the macroscopic hardness with indentation depth is investigated as a function in contact length. The study is conducted for both a nearly flat wedge and a $90^{\circ}$ wedge. The main conclusions are;

- The gradient based model, and the associated strain gradient hardening, results in smeared-out plastic slip fields compared to the conventional plasticity model predictions, with increasingly smooth transitions between different slip regions, for increasing length parameter, $L_{D}$. Moreover, the GND density is predicted to be lower and to accumulate further away from the indentation tip when employing the gradient based model. This is tied to the extra cost in terms of energy for developing plastic slip gradients. Thus, the effect is most significant at the smallest scales of indentation (high value of $L_{D} / a_{c}$ ), and the cost of plastic slip gradients becomes less influential for large indentations where the strain gradient based model predictions eventually converges toward conventional plasticity predictions.

- The gradient based model captures the trend in terms of size dependence of hardness at small scales observed in experiments. The increase in hardness is linked to the predicted changes in GND density fields, where lower densities are predicted for small indentations as the material offers increased plastic resistance due to the energetic cost of developing large GND densities. Hence, it also naturally follows that the effect of slip gradient hardening is further amplified for the $90^{\circ}$ indenter relative to the flat indenter as larger slip gradients (and thereby larger GND densities) are needed to accommodate the deformation imposed on the material around the indenter.

- For the $90^{\circ}$ indenter, pile-up is predicted throughout the entire indentation process for conventional plasticity as well as for the lower considered length scales. However, for $L_{D} / a_{c}^{0}=4$, sink-in is predicted in the beginning of the indentation, which later evolves into pile-up as the indentation proceeds. For the length parameter of $L_{D} / a_{c}^{0}=16$ this transition occurred when the contact length reaches approximately $a_{c} / a_{c}^{0}=7$, and it is caused by the relative decrease of the gradient hardening with increasing indentation. It is expected that the shiift from sink-in to pile-up could be captured in an experimental setup on sufficient small scales.

- For the nearly flat wedge, the plastic slip and GND densities are dominated by effective slip system (1) and (3) at all scales (though most sig- 
nificant at the smallest scales). In contrast, slip system (2) has significant contributions to the GND densities for the $90^{\circ}$ indenter at all scales.

\section{Acknowledgments}

KLN and CFN are financially supported by The Danish Council for Independent Research in the project "New Advances in Steady-State Engineering Techniques", grant: DFF-4184-00132.

A. Acharya and J.L. Bassani. Lattice incompatibility and a gradient theory of crystal plasticity. Journal of the Mechanics and Physics of Solids, 48:1565$1595,2000$.

M.F. Ashby. The deformation of plastically non-homogeneous alloys. Philosophical Magazine, 21:399-424, 1970.

L. Bardella. A deformation theory of strain gradient crystal plasticity that accounts for geometrically necessary dislocations. Journal of the Mechanics and Physics of Solids, 54:128-160, 2006a.

L. Bardella. Some remarks on the strain gradient crystal plasticity modelling, with particular reference to the material length scales involved. International Journal of Plasticity, 23:296-322, 2006b.

U. Borg. A strain gradient crystal plasticity analysis of grain size effects in polycrystals. European Journal of Mechanics/A Solids, 26:313-324, 2007.

L.P. Evers, W.A.M. Brekelmans, and M.G.D. Geers. Non-local crystal plasticity model with intrinsic ssd and gnd effects. Journal of the Mechanics and Physics of Solids, 52:2379-2401, 2004.

N.A. Fleck and J.W. Hutchinson. Strain gradient plasticity. Advances in Applied Mechanics, 33(C):295-361, 1997.

N.A. Fleck and J.W. Hutchinson. A reformulation of strain gradient plasticity. Journal of Mechanics and Physics of Solids, 49:2245-2271, 2001.

N.A. Fleck and J.R. Willis. A mathematical basis for strain.gradient plasticity theory. part ii: tensorial plastic multiplier. Journal of the Mechanics and Physics of Solids, 57:1045-1057, 2009.

N.A. Fleck, G.M. Muller, M.F. Ashby, and J.W. Hutchinson. Strain gradient plasticity: Theory and experiment. Acta metall. mater, 42:475-487, 1994.

H. Gao, Y. Huang, W.D. Nix, and J.W. Hutchinson. Mechanism-based strain gradient plasticity - i. theory. Journal of the Mechanics and Physics of Solids, 47(6):1239-1263, 1999.

P. Gudmundson. A united treatment of strain gradient plasticity. Journal of the Mechanics and Physics of Solids, 52:1379 1406, 2004. 
M.E. Gurtin. A gradient theory of single-crystal viscoplasticity that accounts for geometrically necessary dislocations. Journal of the Mechanics and Physics of Solids, 50:5-32, 2002.

M.E. Gurtin, L. Anand, and S.P. Lele. Gradient single-crystal plasticity with free energy dependent on dislocation densities. Journal of the Mechanics and Physics of Solids., 55:1853-1878, 2007.

R. Hill. Some basic principles in the mechanics of solids without a natural time. Journal of the Mechanics and Physics of Solids, 7:209-225, 1959.

Y. Huang, S. Qu, Hwang K.C., M. Li, and H. Gao. A conventional theory of mechanism-based strain gradient plasticity. International Journal of Plasticity, 20:753-782, 2000.

K. J. Juul, C. Nellemann, K. L. Nielsen, C. F. Niordson, and J. W. Kysar. Wedge indentation of single crystalline monazite: A numerical investigation. Submitted for publication, 2018a.

K. J. Juul, C. F. Niordson, K. L. Nielsen, and J. W. Kysar. A novel numerical framework for self-similarity in plasticity: Wedge indentation in single crystals. Journal of the Mechanics and Physics of Solids, 112:667-684, 2018b. doi: 10.1016/j.jmps.2017.09.011.

M. Kuroda and V. Tvergaard. Studies of scale dependent crystal viscopalsticity models. Journal of the Mechanics and Physics of Solids, 54:1789-1810, 2006.

Jeffrey W. Kysar and Yong X. Gan. Void growth in fcc and bcc single crystals. 2016. doi: 10.1.1.915.8625.

Jeffrey W. Kysar, Yong X. Gan, Timothy L. Morse, Xi Chen, and Milton E. Jones. High strain gradient plasticity associated with wedge indentation into face-centered cubic single crystals: Geometrically necessary dislocation densities. Journal of the Mechanics and Physics of Solids, 55(7):1554-1573, 2007. ISSN 18734782, 00225096. doi: 10.1016/j.jmps.2006.09.009.

M.J. Lewandowski and S. Stupkiewicz. Size effects in wedge indentation predicted by a gradient-enhanced crystal-plasticity model. International Journal of Plasticity, 2018. ISSN 18792154, 07496419. doi: 10.1016/j.ijplas.2018.05.008.

Q. Ma and D.R. Clarke. Size dependent hardness of silver single crystals. Journal of Materials Research, 10 issue 4:853-863, 1995.

K.W. McElhaney, J.J. Vlassak, and W.D. Nix. Determination of indenter til geometry and indentation contact area for depth-sensing indentation experiments. Journal of materials research, 13:1300-1306, 1997.

R.M. McMeeking and J.R. Rice. Finite clement formulations for problems of large elastic-plastic deformation. International Journal of Solids and Structures, 11:601-616, 1975. 
C.F. Niordson and J.W. Kysar. Computational strain gradient crystal plasticity. Journal of the Mechanicsand Physics of Solids., 62:31-47, 2013.

C.F. Niordson and P. Redanz. Size-effects in plane strain sheet-necking. Journal of the Mechanics and Physics of Solids, 52:2431-2454, 2004.

D. Peirce, R.J. Asaro, and A. Needleman. Material rate dependence and localized deformation in crystalline solids. Acta Metallurgica, 31:19511976, 1983.

J.R. Rice. Tensile crack tip fields in elastic-ideally plastic crystal. Mechanics of Materials, 6:317-335, 1987.

N.A. Stelmashenko, M.G. Walls, L.M. Brown, and Y.V. Milman. Microindentations on W and Mo oriented single crystals: An STM study. Acta Metallurgica et Materialia, 41:2855-2865, 1993.

Y. Zhang, Y. Gao, and L. Nicola. Lattice rotation caused by wedge indentation of a single crystal: Dislocation dynamics compared to crystal plasticity simulations. Journal of the Mechanics and Physics of Solids, 68:267-279, 2014. 


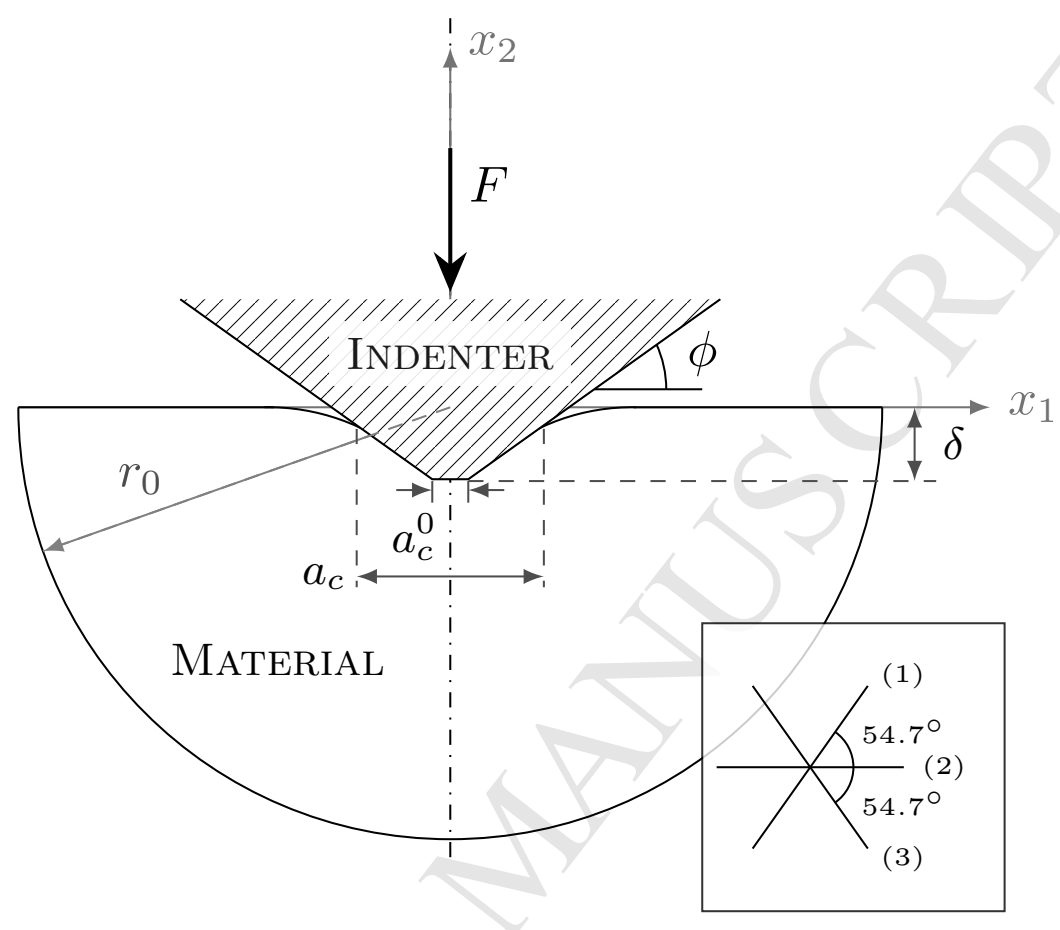

Figure 1: Schematic of the problem, where $\delta$ is the indentation depth, $\phi$ is the angle between the wedge sides and horizontal, $a_{c}^{0}$ is the initial flat contact length, $a_{c}$ is the contact length in the deformed configuration (here for a sink-in indentation), $r_{0}$ is the domain radius, and $F$ is the reaction force between the indenter and the domain. The domain is of unit thickness, $t$. Bottom right is the direction of the three effective in-plane slip systems $(\alpha)$ illustrated. 


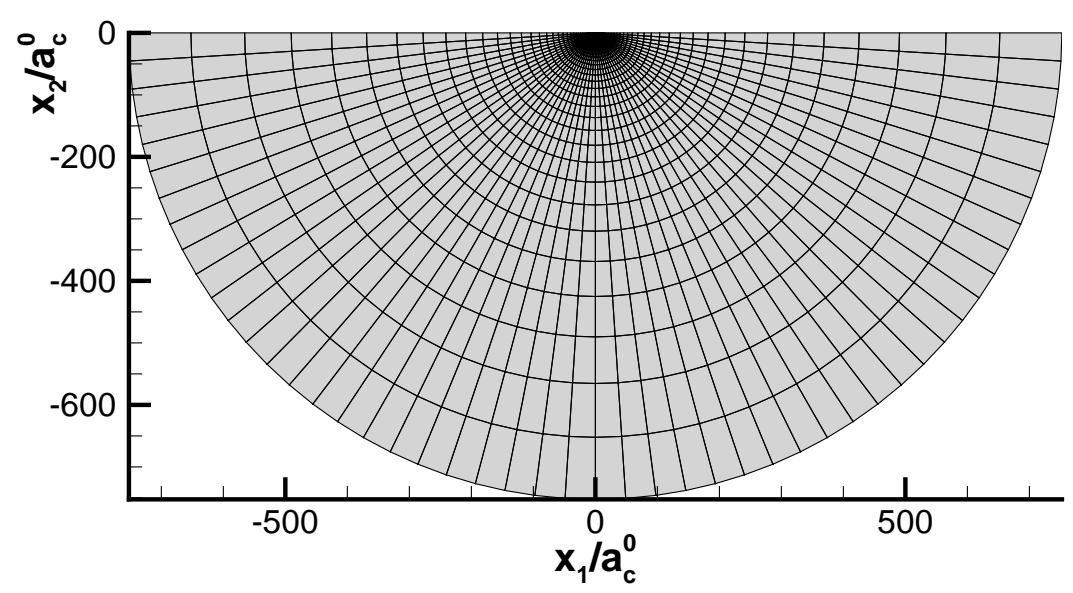

(a)

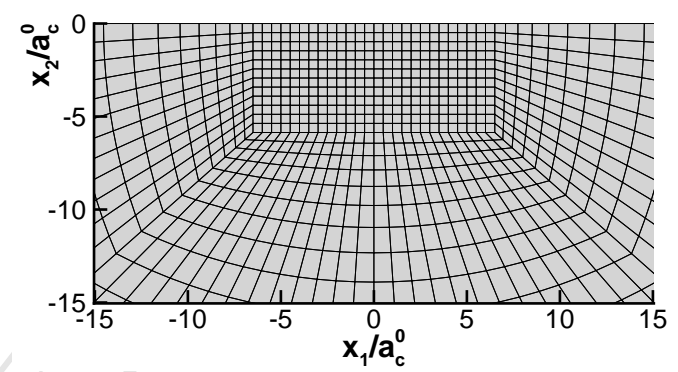

(b)

Figure 2: Employed Finite Elemenet mesh with 50 elements throughout the domain radius, 50 elements along the periphery and a factor of 200 in height difference between the smallest and largest elements. (a) in full size and (b) $50 \times$ zoom around $\left(x_{1}, x_{2}\right)=(0,0)$ being the first region in contact with the indenter. 


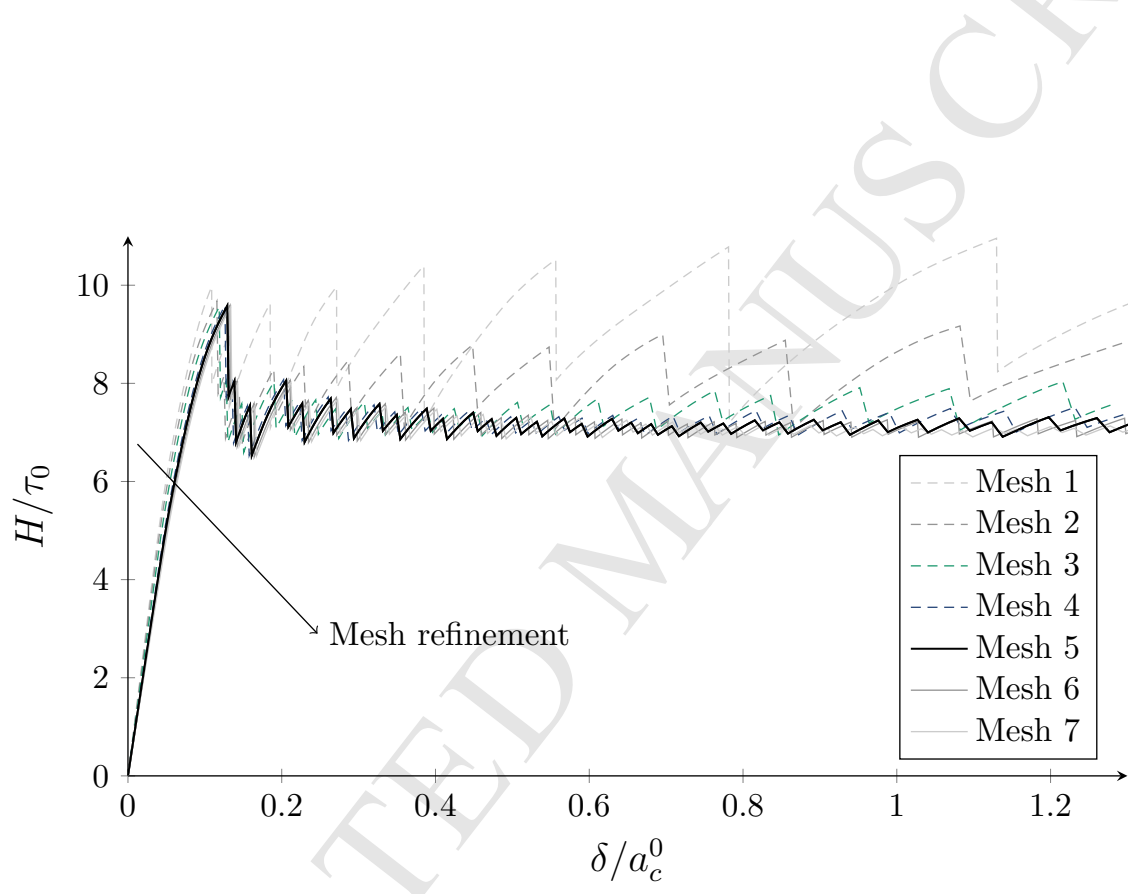

Figure 3: Mesh convergence of hardness curves showing the hardness as a function of the indentation depth for the seven different mesh resolutions listed in Table 3. 


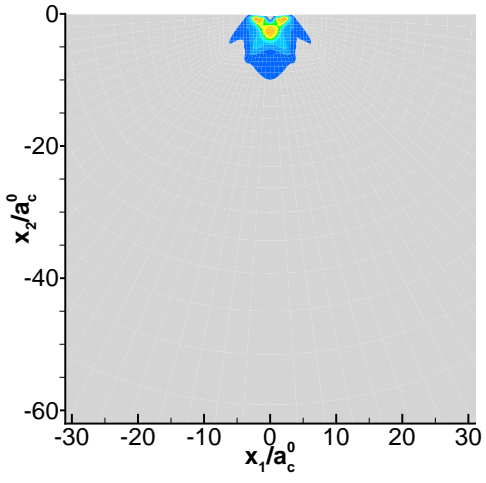

(a) $a_{c} / a_{c}^{0}=3$

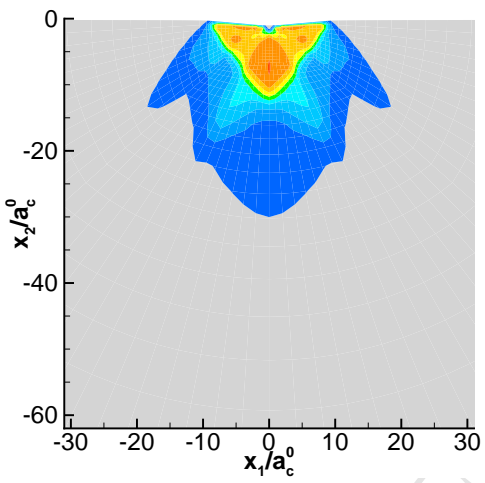

(c) $a_{c} / a_{c}^{0}=9$

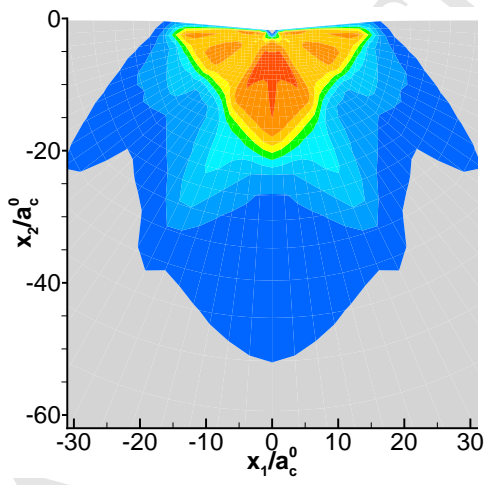

(e) $a_{c} / a_{c}^{0}=15$

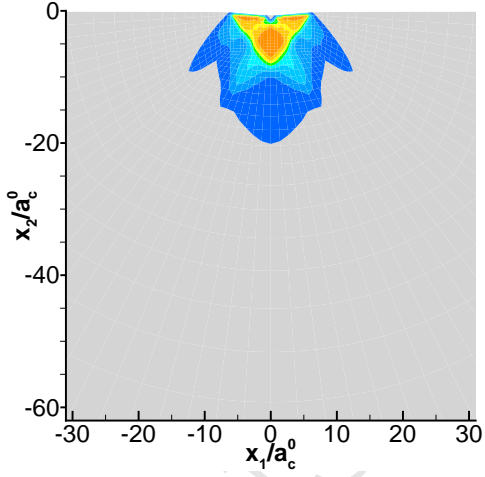

(b) $a_{c} / a_{c}^{0}=6$

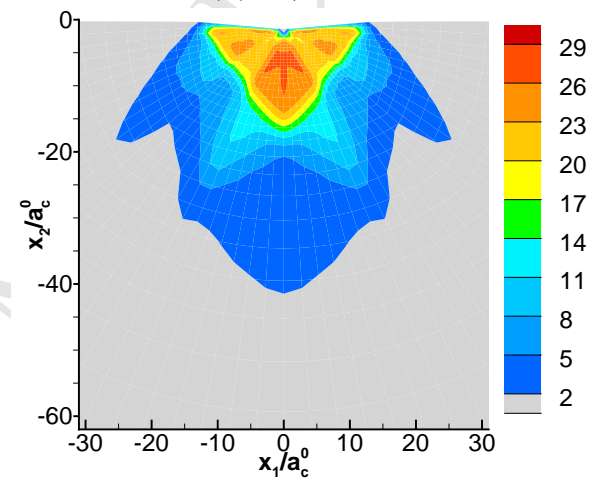

(d) $a_{c} / a_{c}^{0}=12$

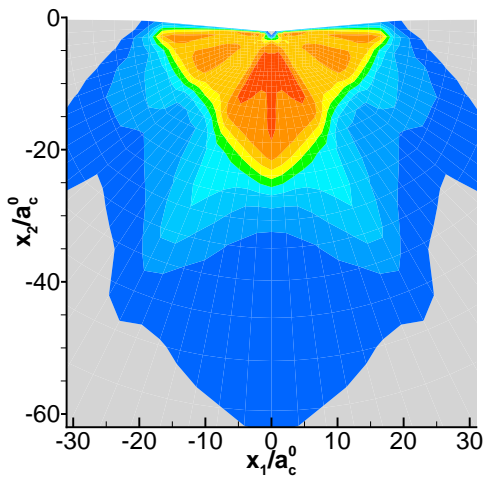

(f) $a_{c} / a_{c}^{0}=18$

Figure 4: Total slip $\gamma^{T} / \gamma_{0}$ for conventional plasticity $\left(L_{D}=0\right)$ at different contact lengths showing a self-similar evolution after a short transient state. 


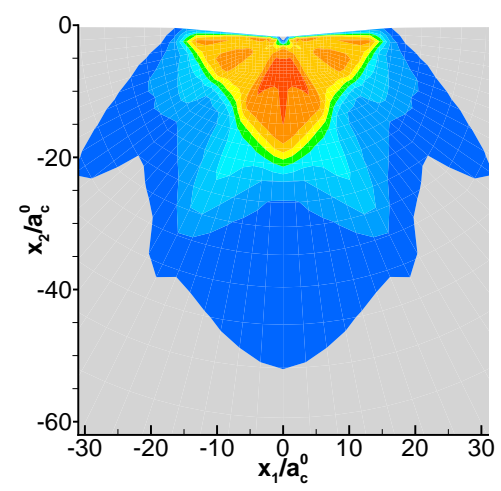

(a) $\gamma^{T} / \gamma_{0}$

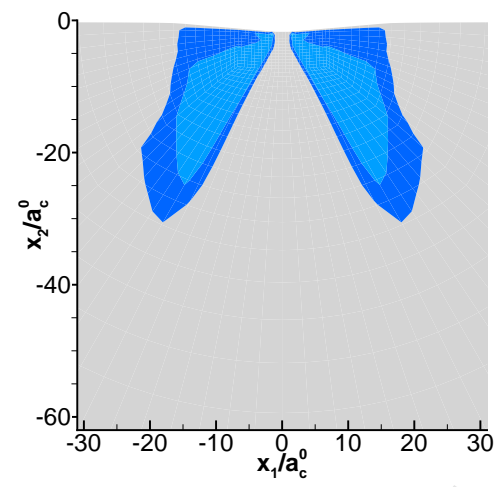

(c) $\left|\gamma^{(2)}\right| / \gamma_{0}^{(2)}$

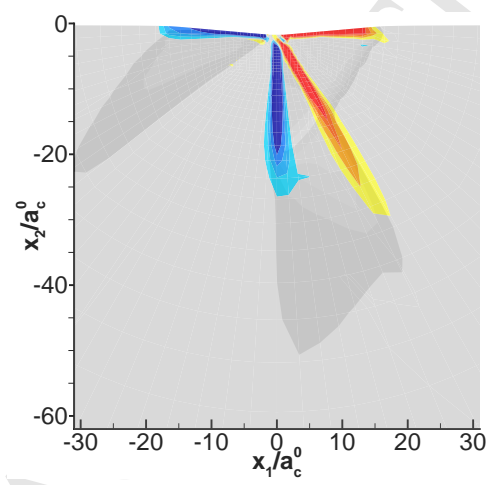

(e) $\rho^{(1)} / \rho_{0}^{(1)}$

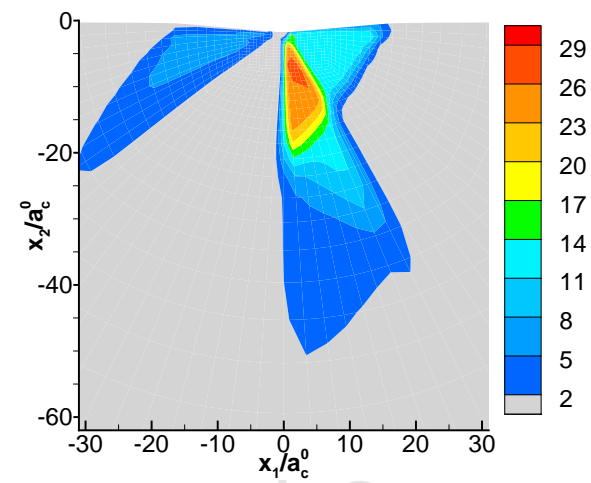

(b) $\left|\gamma^{(1)}\right| / \gamma_{0}^{(1)}$

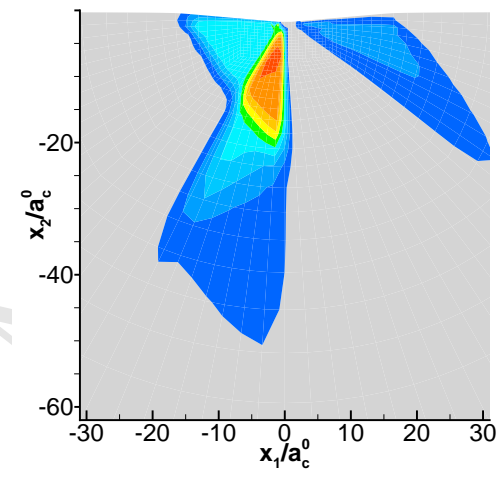

(d) $\left|\gamma^{(3)}\right| / \gamma_{0}^{(3)}$

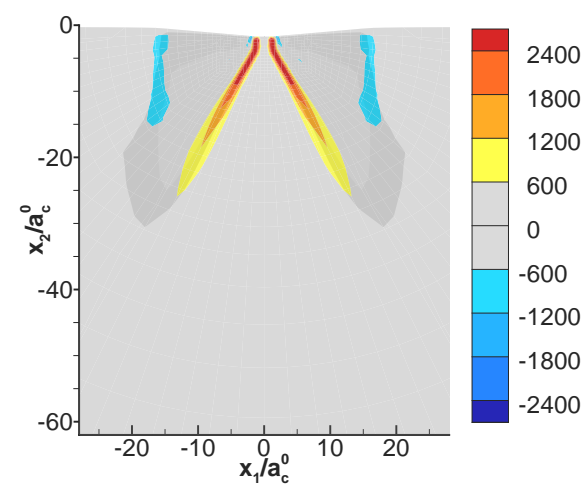

(f) $\rho^{(2)} / \rho_{0}^{(2)}$

Figure 5: Total slip $\gamma^{T} / \gamma_{0}$, individual effective slip $\left|\gamma^{(\alpha)}\right| / \gamma_{0}^{(\alpha)}$ and GND-densities $\rho^{(\alpha)} / \rho_{0}^{(\alpha)}$ (with the individual slip as a faded overlay) for conventional plasticity $\left(L_{D}=0\right)$ at an indentation length $a_{c} / a_{c}^{0}=15$ and with $\phi=5^{\circ}$. 


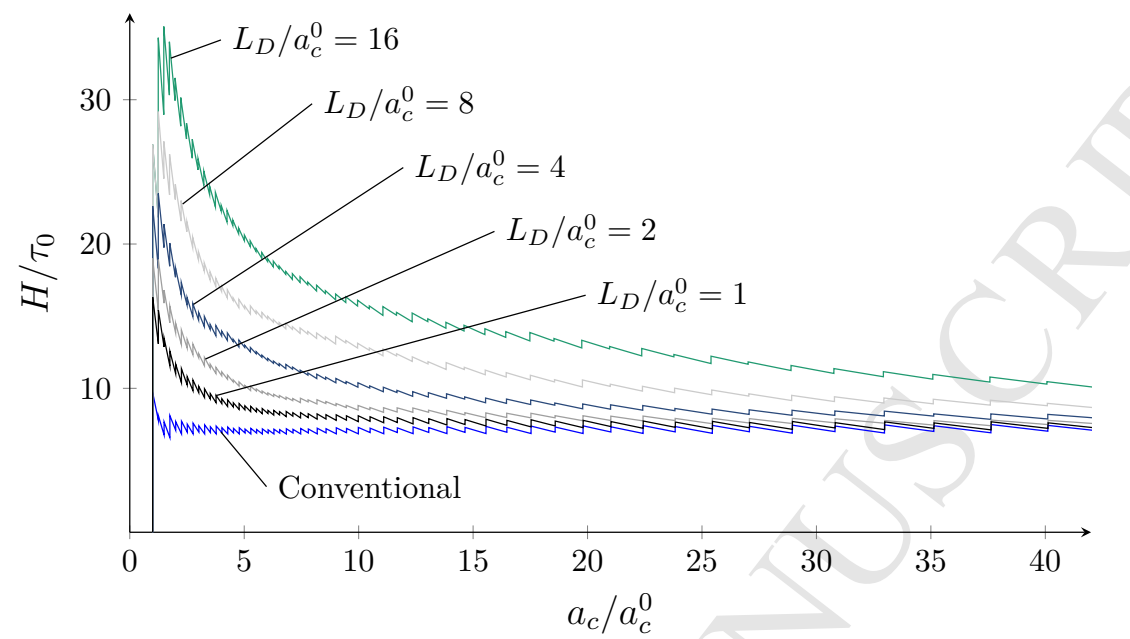

Figure 6: Hardeness curves for different dissipative length parameters $L_{D} / a_{c}^{0}=[1,2,4,8,16]$ and for conventional plasticity $\left(L_{D}=0\right)$ showing size-effects on macroscopic hardness for $\phi=5^{\circ}$.

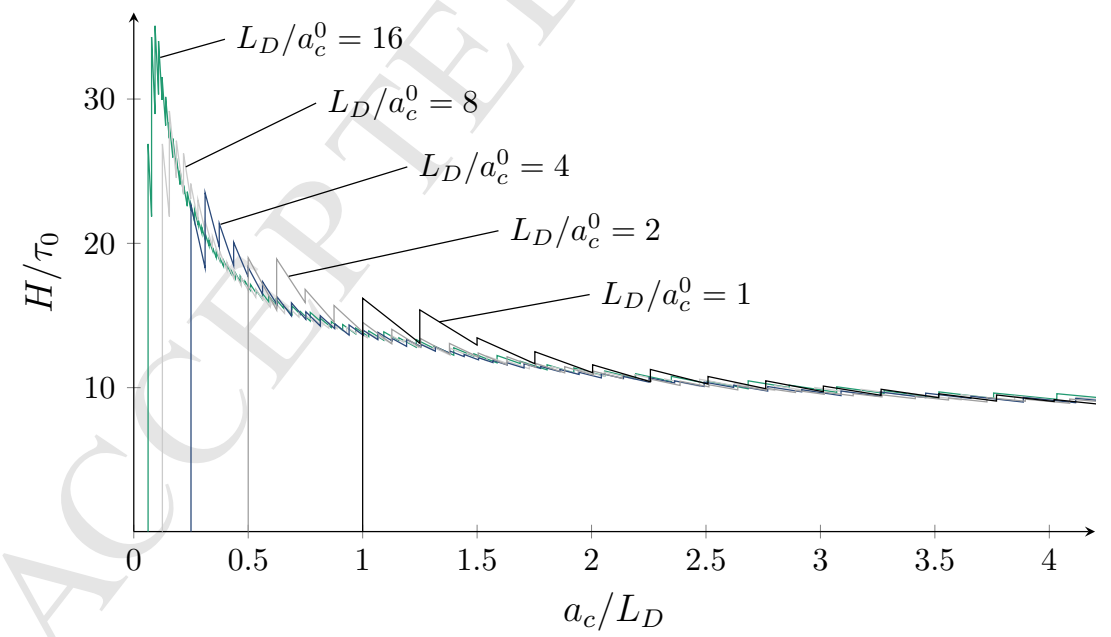

Figure 7: Hardness curves with contact length normalized with respect to the length parameter for $\phi=5^{\circ}$ showing overlapping of the curves. 


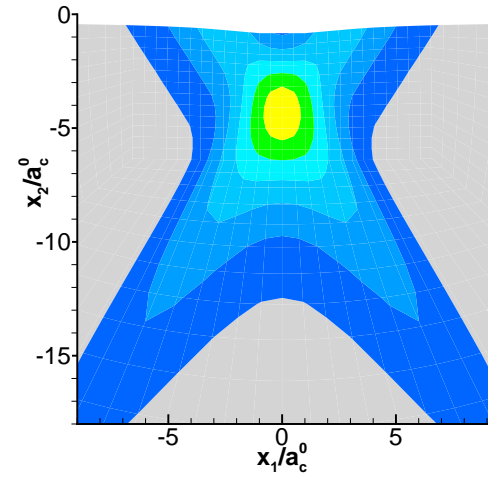

(a) $a_{c} / a_{c}^{0}=3$

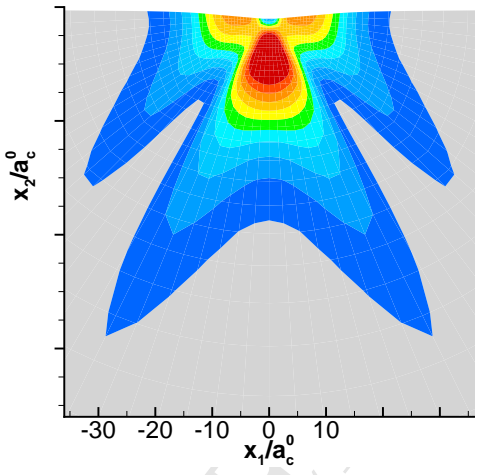

(c) $a_{c} / a_{c}^{0}=12$

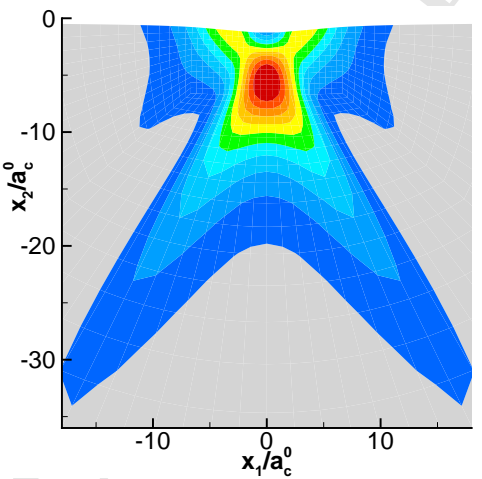

(b) $a_{c} / a_{c}^{0}=6$

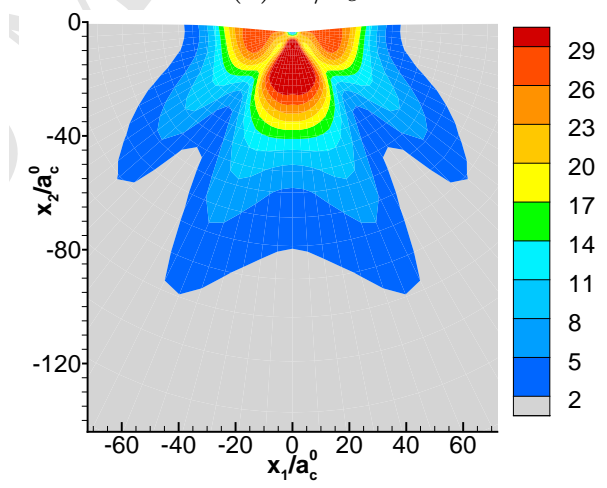

(d) $a_{c} / a_{c}^{0}=24$

Figure 8: Total slip $\gamma^{T} / \gamma_{0}$ at different current contact lengths with a dissipative length parameter of $L_{D} / a_{c}^{0}=16$ and $\phi=5^{\circ}$. 


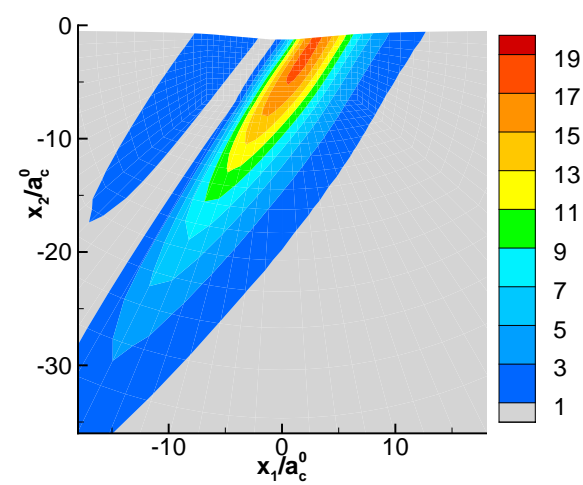

(a) $\left|\gamma^{(1)}\right| / \gamma_{0}^{(1)}$

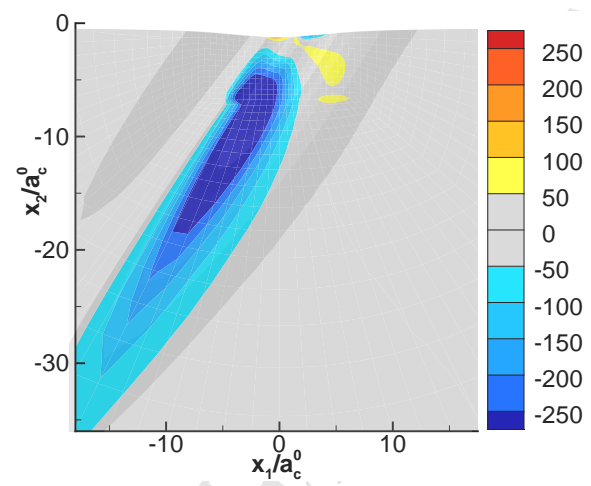

(c) $\rho^{(1)} / \rho_{0}^{(1)}$

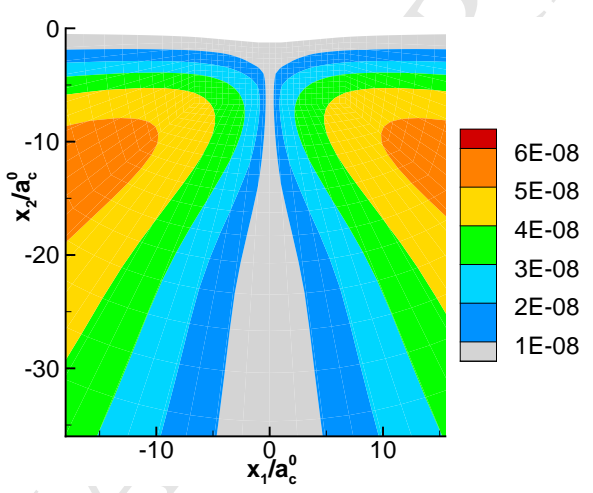

(b) $\left|\gamma^{(2)}\right| / \gamma_{0}^{(2)}$

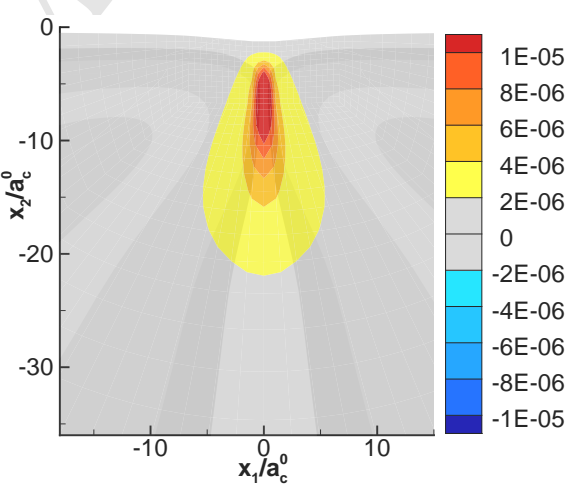

(d) $\rho^{(2)} / \rho_{0}^{(2)}$

Figure 9: Individual effective slip $\left|\gamma^{(\alpha)}\right| / \gamma_{0}^{(\alpha)}$ and GND-densities $\rho^{(\alpha)} / \rho_{0}^{(\alpha)}$ (with the individual slip as a faded overlay) for $a_{c} / a_{c}^{0}=6, L_{D} / a_{c}^{0}=16$ and with $\phi=5^{\circ}$. 


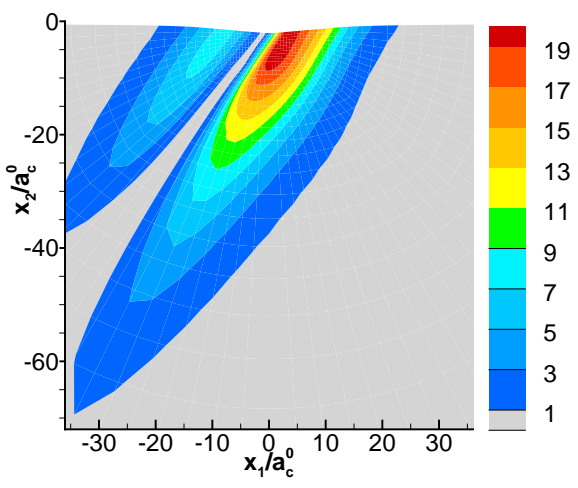

(a) $\left|\gamma^{(1)}\right| / \gamma_{0}^{(1)}$

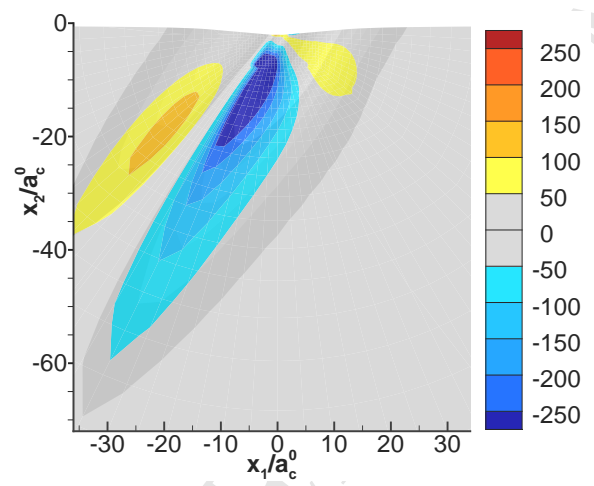

(c) $\rho^{(1)} / \rho_{0}^{(1)}$

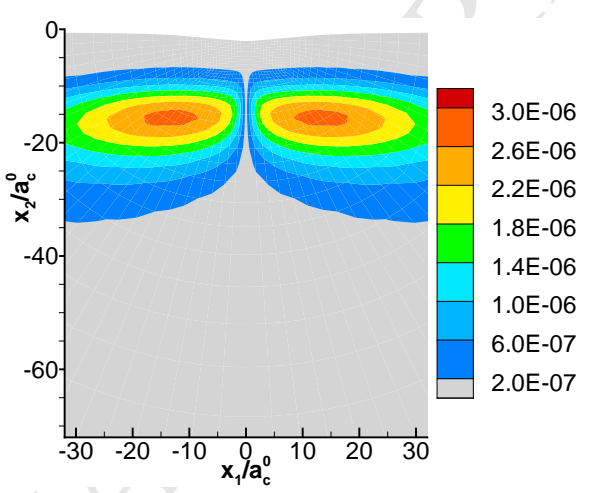

(b) $\left|\gamma^{(2)}\right| / \gamma_{0}^{(2)}$

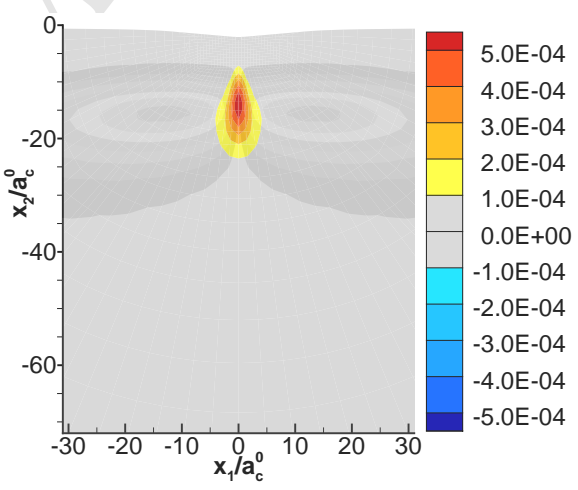

(d) $\rho^{(2)} / \rho_{0}^{(2)}$

Figure 10: Individual effective slip $\left|\gamma^{(\alpha)}\right| / \gamma_{0}^{(\alpha)}$ and GND-densities $\rho^{(\alpha)} / \rho_{0}^{(\alpha)}$ (with the individual slip as a faded overlay) for $a_{c} / a_{c}^{0}=12, L_{D} / a_{c}^{0}=16$ and with $\phi=5^{\circ}$. 


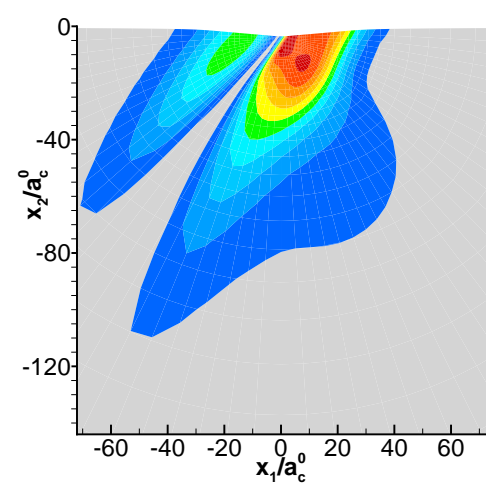

(a) $\left|\gamma^{(1)}\right| / \gamma_{0}^{(1)}$

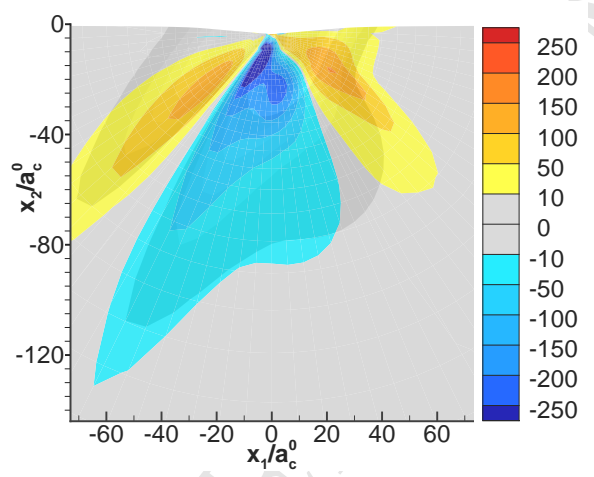

$$
\text { (c) } \rho^{(1)} / \rho_{0}^{(1)}
$$

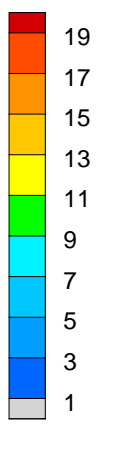

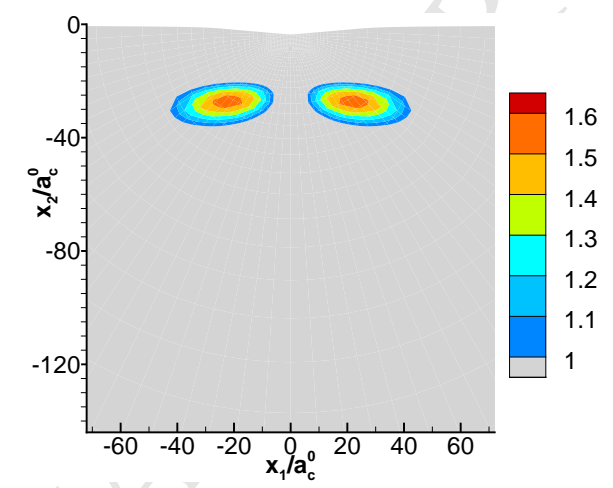

(b) $\left|\gamma^{(2)}\right| / \gamma_{0}^{(2)}$

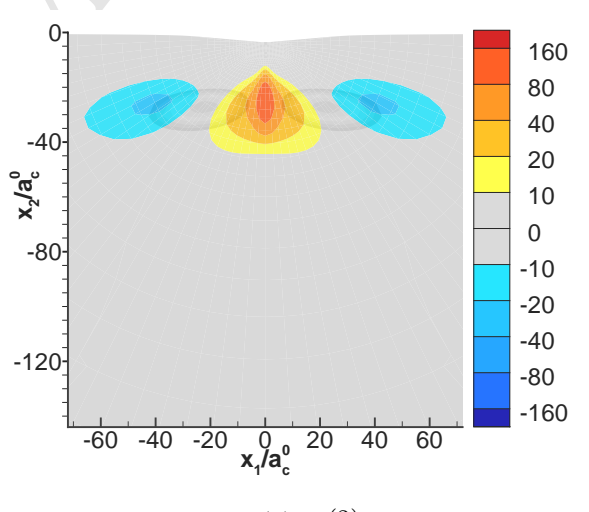

(d) $\rho^{(2)} / \rho_{0}^{(2)}$

Figure 11: Individual effective slip $\left|\gamma^{(\alpha)}\right| / \gamma_{0}^{(\alpha)}$ and GND-densities $\rho^{(\alpha)} / \rho_{0}^{(\alpha)}$ (with the individual slip as a faded overlay) for $a_{c} / a_{c}^{0}=24, L_{D} / a_{c}^{0}=16$ and with $\phi=5^{\circ}$. 


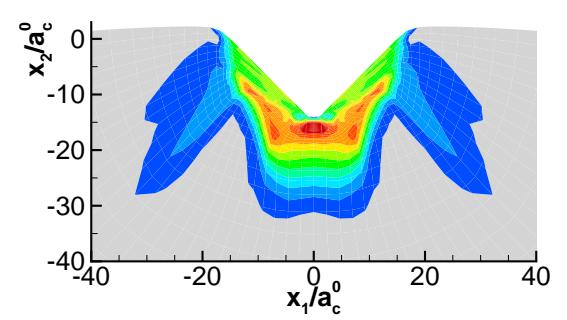

(a) $\gamma^{(T)} / \gamma_{0}$

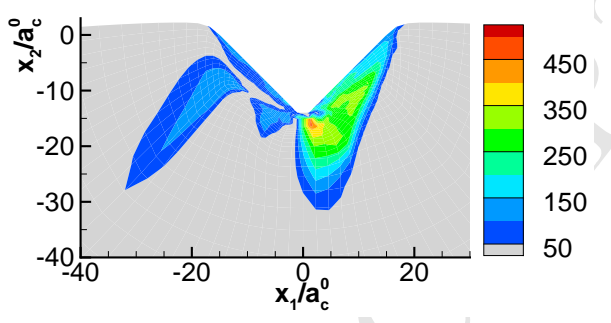

(b) $\left|\gamma^{(1)}\right| / \gamma_{0}^{(1)}$

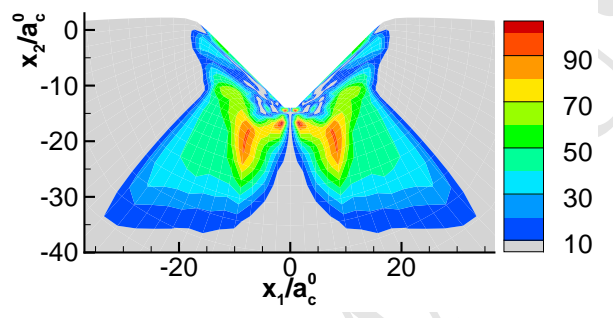

(c) $\left|\gamma^{(2)}\right| / \gamma_{0}^{(2)}$

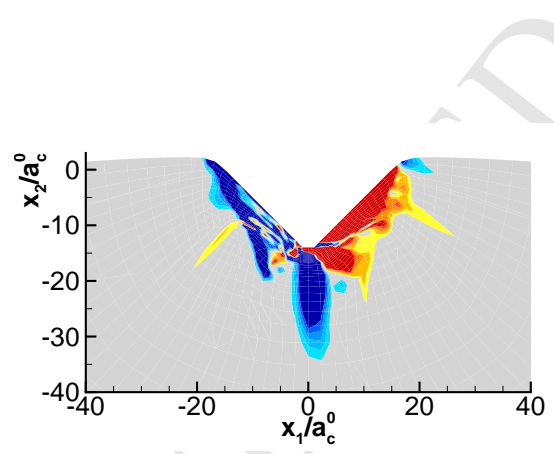

(d) $\rho^{(1)} / \rho_{0}^{(1)}$

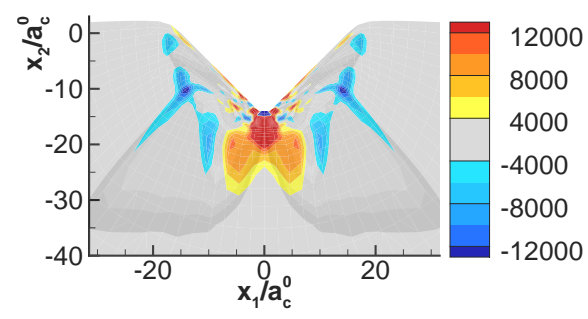

(e) $\rho^{(2)} / \rho_{0}^{(2)}$

Figure 12: Total slip $\gamma^{T} / \gamma_{0}$, individual effective slip $\left|\gamma^{(\alpha)}\right| / \gamma_{0}^{(\alpha)}$ and GND-densities $\rho^{(\alpha)} / \rho_{0}^{(\alpha)}$ (with the individual slip as a faded overlay) for conventional plasticity at contact length $a_{c} / a_{c}^{0}=15$ and with $\phi=45^{\circ}$. 


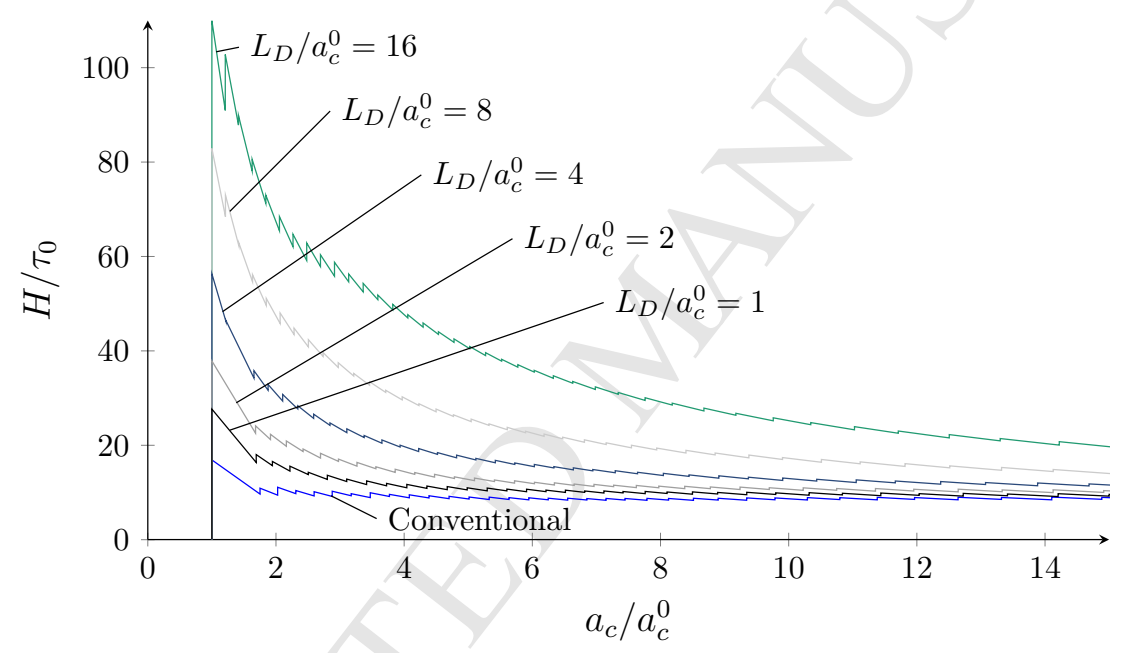

Figure 13: Hardness curves for the dissipative length parameters $L_{D} / a_{c}^{0}=[1,2,4,8,16]$ and for conventional plasticity $\left(L_{D}=0\right)$ showing size-effects on macroscopic hardening for $\phi=$ $45^{\circ}$. 


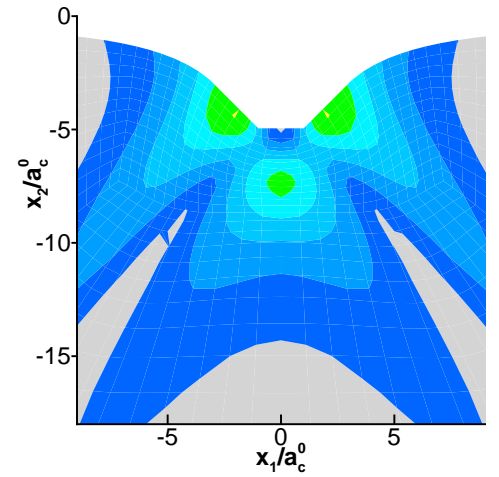

(a) $a_{c} / a_{c}^{0}=3$

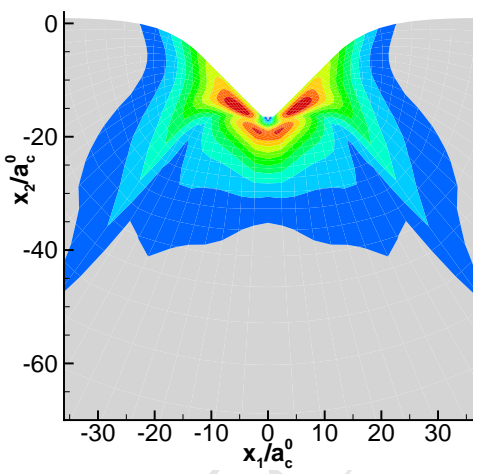

(c) $a_{c} / a_{c}^{0}=12$

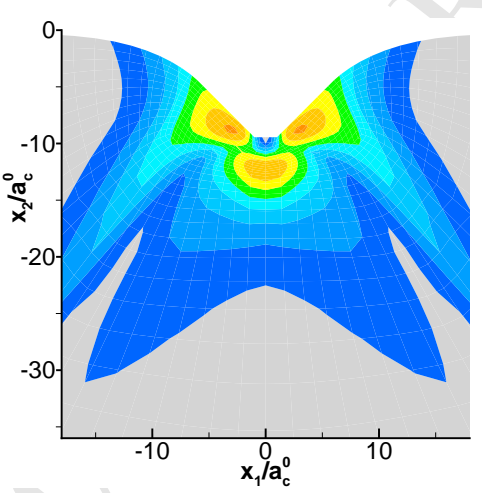

(b) $a_{c} / a_{c}^{0}=6$

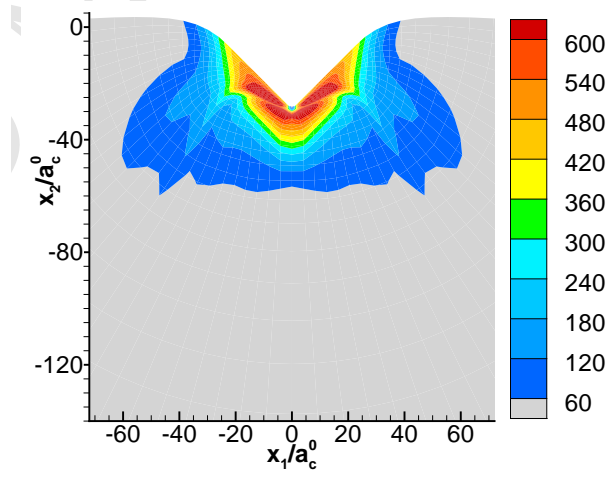

(d) $a_{c} / a_{c}^{0}=24$

Figure 14: Total slip $\gamma^{T} / \gamma_{0}$ at different contact lengths with a dissipative length parameter of $L_{D} / a_{c}^{0}=16$ and $\phi=45^{\circ}$. 

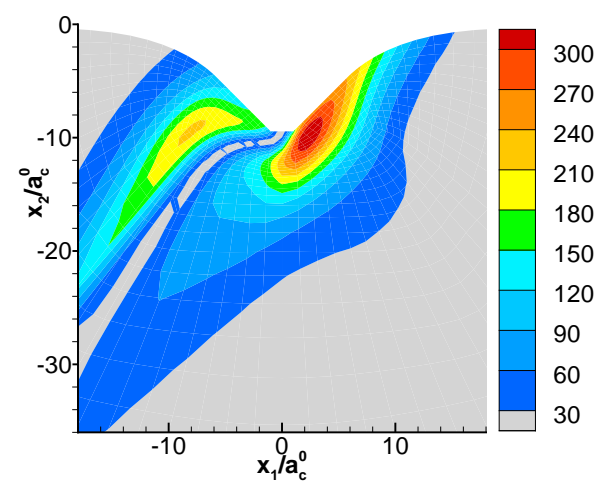

(a) $\left|\gamma^{(1)}\right| / \gamma_{0}^{(1)}$

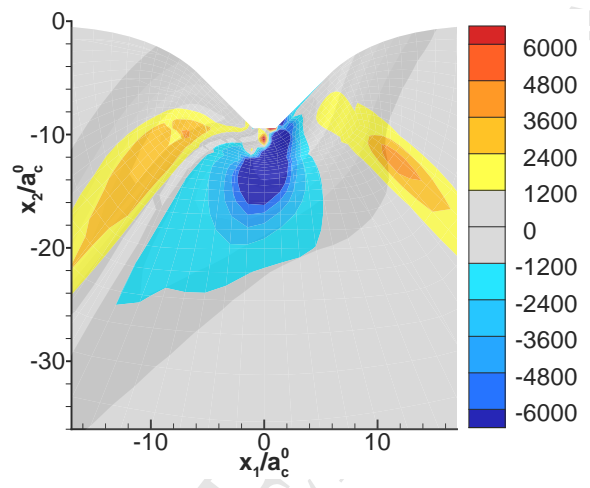

(c) $\rho^{(1)} / \rho_{0}^{(1)}$

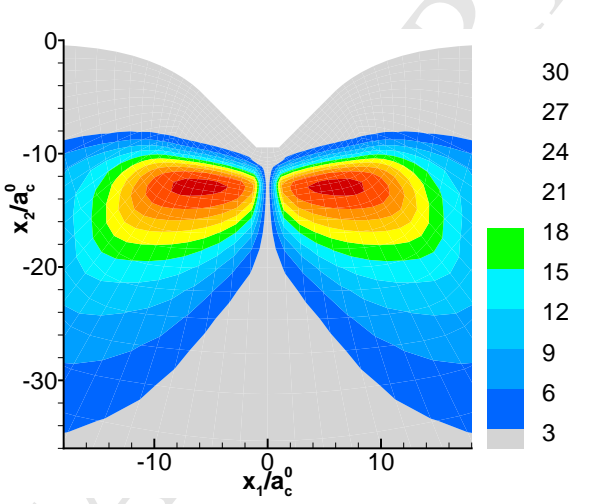

(b) $\left|\gamma^{(2)}\right| / \gamma_{0}^{(2)}$

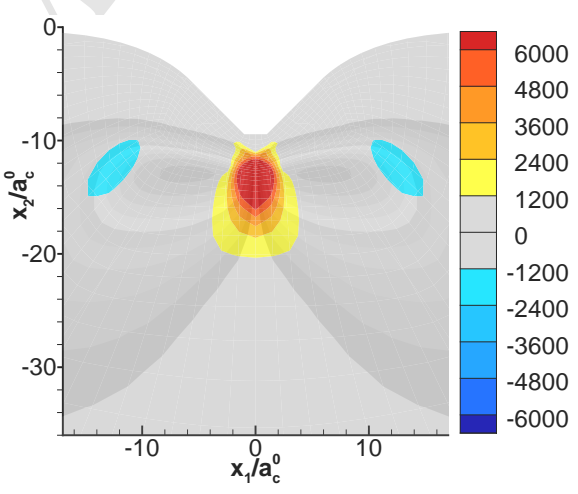

(d) $\rho^{(2)} / \rho_{0}^{(2)}$

Figure 15: Individual effective slip $\left|\gamma^{(\alpha)}\right| / \gamma_{0}^{(\alpha)}$ and GND-densities $\rho^{(\alpha)} / \rho_{0}^{(\alpha)}$ (with the individual slip as a faded overlay) for $a_{c} / a_{c}^{0}=6, L_{D} / a_{c}^{0}=16$ and with $\phi=45^{\circ}$. 


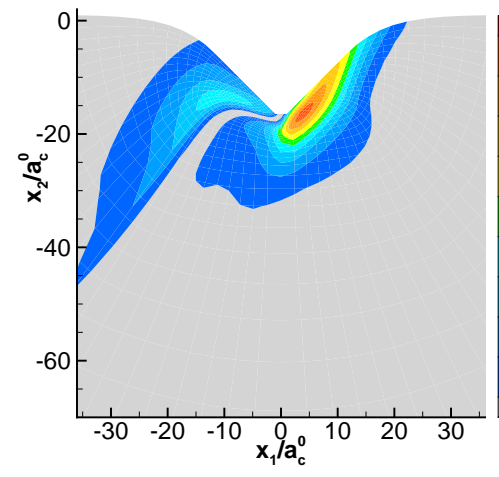

(a) $\left|\gamma^{(1)}\right| / \gamma_{0}^{(1)}$

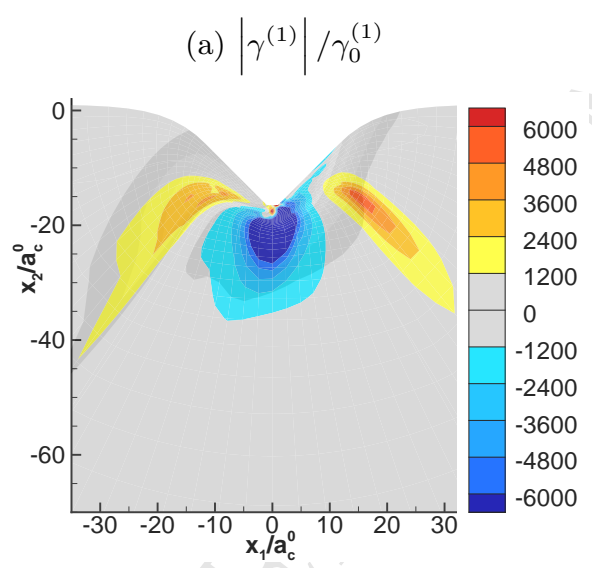

$$
\text { (c) } \rho^{(1)} / \rho_{0}^{(1)}
$$

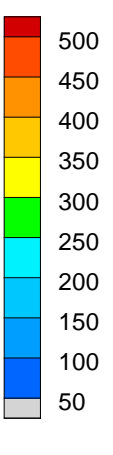

4

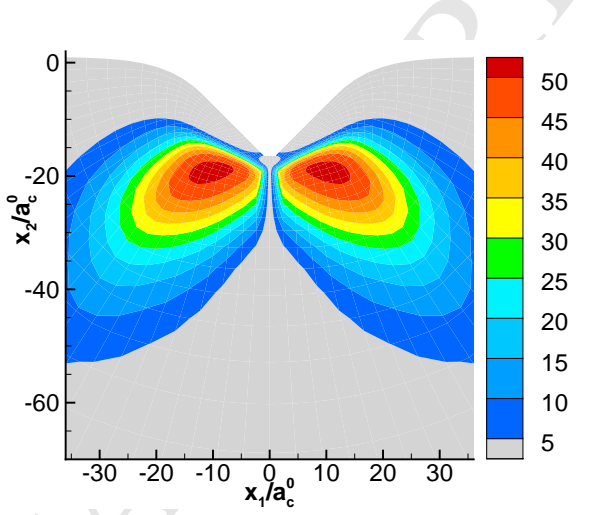

(b) $\left|\gamma^{(2)}\right| / \gamma_{0}^{(2)}$

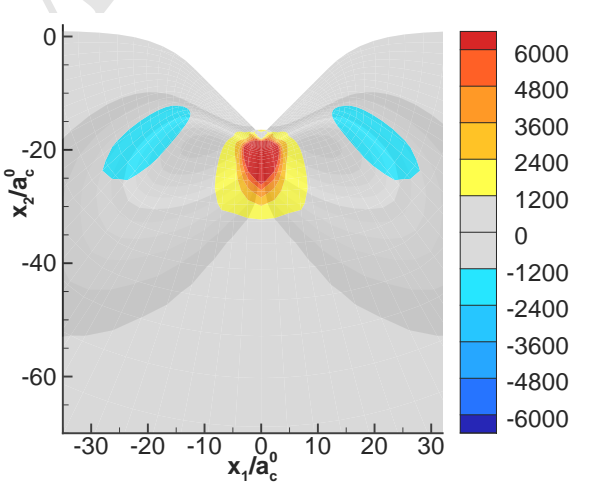

(d) $\rho^{(2)} / \rho_{0}^{(2)}$

Figure 16: Individual effective slip $\left|\gamma^{(\alpha)}\right| / \gamma_{0}^{(\alpha)}$ and GND-densities $\rho^{(\alpha)} / \rho_{0}^{(\alpha)}$ (with the individual slip as a faded overlay) for $a_{c} / a_{c}^{0}=12, L_{D} / a_{c}^{0}=16$ and with $\phi=45^{\circ}$. 


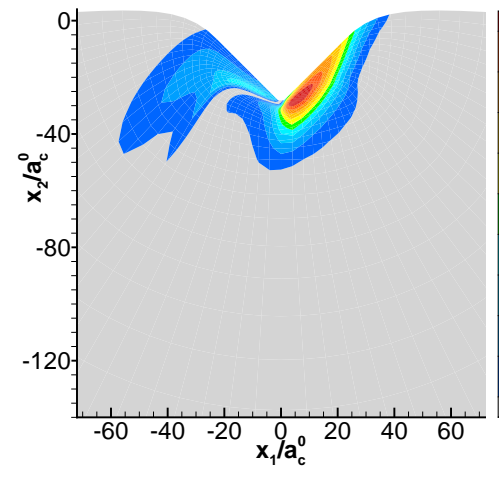

(a) $\left|\gamma^{(1)}\right| / \gamma_{0}^{(1)}$

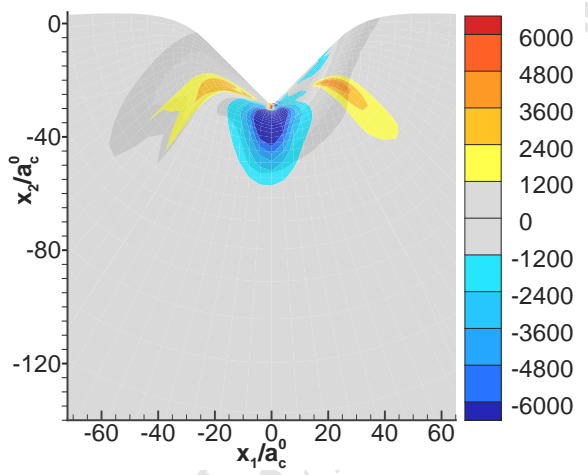

(c) $\rho^{(1)} / \rho_{0}^{(1)}$

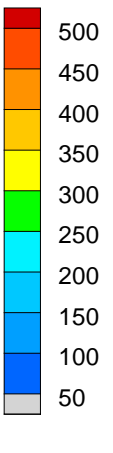

6000
4800
3600
2400
1200
0
-1200
-2400
-3600
-4800
-6000

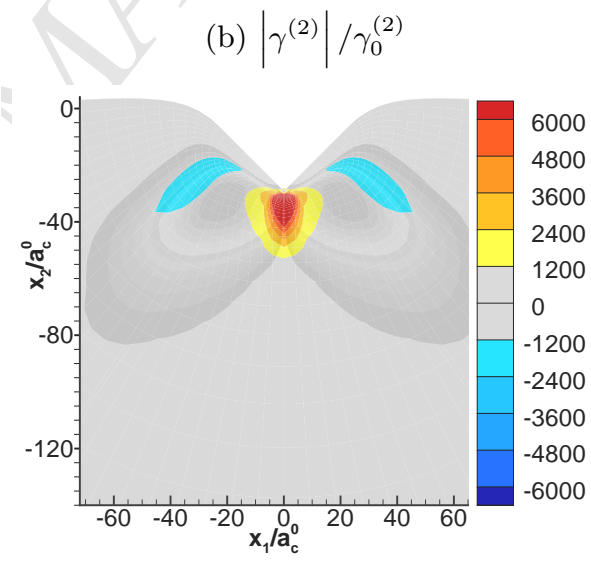

(d) $\rho^{(2)} / \rho_{0}^{(2)}$

Figure 17: Individual effective slip $\left|\gamma^{(\alpha)}\right| / \gamma_{0}^{(\alpha)}$ and GND-densities $\rho^{(\alpha)} / \rho_{0}^{(\alpha)}$ (with the individual slip as a faded overlay) for $a_{c} / a_{c}^{0}=24, L_{D} / a_{c}^{0}=16$ and with $\phi=45^{\circ}$. 


\begin{tabular}{c|lcc}
\hline & Parameter & Symbol & Value \\
\hline \multirow{5}{*}{ Geometry } & Domain radius & $r_{0}$ & $1 \mathrm{~mm}$ \\
& Wedge angle & $\phi$ & $5^{\circ}, 45^{\circ}$ \\
& Initial contact length & $a_{c}^{0}$ & $2.659 \mu \mathrm{m}$ \\
\hline \multirow{5}{*}{ Material data } & Youngs modulus & $E$ & $76 \mathrm{GPa}$ \\
& Poissons ratio & $\nu$ & 0.333333 \\
& Initial slip resistance & $\tau_{0}$ & $100 \mathrm{MPa}$ \\
& Reference slip rate & $\dot{\gamma}_{0}$ & $10^{-3} \mathrm{~s}^{-1}$ \\
& The visco-plastic exponent & $m$ & 0.02 \\
& Indentation rate & $\dot{\varepsilon}_{v}$ & $4 \cdot 10^{-3} s^{-1}$ \\
& Dissipative length parameter & $L_{D} / a_{c}^{0}$ & $0,1,2,4,8,16$ \\
\hline
\end{tabular}

Table 1: Model input parameters. 


\begin{tabular}{l|ccc}
\hline Effective slip system $(\alpha)$ & $(1)$ & $(2)$ & $(3)$ \\
\hline Angle to $x_{1}$-axis & $54.7^{\circ}$ & $0^{\circ}$ & $-54.7^{\circ}$ \\
Reference effective slip system tensor $s_{i 0}^{(\alpha)}$ & $\frac{\sqrt{3}}{3}\left\{\begin{array}{c}1 \\
\sqrt{2} \\
0\end{array}\right\}$ & $\left\{\begin{array}{l}1 \\
0 \\
0\end{array}\right\}$ & $\frac{\sqrt{3}}{3}\left\{\begin{array}{c}1 \\
-\sqrt{2} \\
0\end{array}\right\}$ \\
Reference effective slip plane normal $m_{i 0}^{(\alpha)}$ & $\frac{\sqrt{3}}{3}\left\{\begin{array}{c}-\sqrt{2} \\
1 \\
0\end{array}\right\}$ & $\left\{\begin{array}{c}0 \\
1 \\
0\end{array}\right\}$ & $\frac{\sqrt{3}}{3}\left\{\begin{array}{c}\sqrt{2} \\
1 \\
0\end{array}\right\}$ \\
Reference crystallograhic slip system tensor $s_{i 0}^{(\alpha a)}$ & $\frac{1}{2}\left\{\begin{array}{c}1 \\
\sqrt{2} \\
-1\end{array}\right\}$ & $\left\{\begin{array}{l}1 \\
0 \\
0\end{array}\right\}$ & $\frac{1}{2}\left\{\begin{array}{c}1 \\
-\sqrt{2} \\
-1\end{array}\right\}$ \\
Reference crystallographic slip plane normal $m_{i 0}^{(\alpha a)}$ & $\frac{1}{3}\left\{\begin{array}{c}-\sqrt{6} \\
\sqrt{3} \\
0\end{array}\right\}$ & $\frac{\sqrt{3}}{3}\left\{\begin{array}{c}0 \\
1 \\
\sqrt{2}\end{array}\right\}$ & $\frac{1}{3}\left\{\begin{array}{c}\sqrt{3} \\
0\end{array}\right\}$ \\
Reference crystallographic slip system tensor $s_{i 0}^{(\alpha b)}$ & $\frac{1}{2}\left\{\begin{array}{c}1 \\
\sqrt{2} \\
-1\end{array}\right\}$ & $s_{i 0}^{(2 a)}$ & $\frac{1}{2}\left\{\begin{array}{c}1 \\
-\sqrt{2} \\
1\end{array}\right\}$ \\
Reference crystallographic slip plane normal $m_{i 0}^{(\alpha b)}$ & $\frac{1}{3}\left\{\begin{array}{c}-\sqrt{6} \\
\sqrt{3} \\
0\end{array}\right\}$ & $\frac{\sqrt{3}}{3}\left\{\begin{array}{c}0 \\
1 \\
-\sqrt{2}\end{array}\right\}$ & $m_{i 0}^{(2 a)}$ \\
$\beta^{(\alpha)}=\frac{S_{i 0}^{(\alpha a)} m_{j 0}^{(\alpha a)}+s_{i 0}^{(\alpha b)} m_{j 0}^{(\alpha b)}}{s_{i 0}^{(\alpha)} m_{j 0}^{(\alpha)}}=\frac{\dot{\gamma}^{(\alpha)}}{\dot{\gamma}^{(\alpha a)}}$ & $\frac{2 \sqrt{3}}{3}$ & $\sqrt{3}$ \\
$\lambda^{(\alpha)}=\frac{\tau^{(\alpha)}}{\tau^{(\alpha a)}}=\frac{\tau^{(\alpha)}}{\tau^{(\alpha b)}=\frac{2}{\beta^{(\alpha)}}}$ & $\frac{2 \sqrt{3}}{3}$ & $\sqrt{3}$ & $\frac{2 \sqrt{3}}{3}$ \\
$\mu^{(\alpha)}=\frac{L_{D}^{(\alpha)}}{L_{D}}=s_{i 0}^{(\alpha a)} s_{i 0}^{(\alpha)}$ & $\frac{\sqrt{3}}{2}$ & $\sqrt{3}$ & $\frac{\sqrt{3}}{2}$ \\
\hline
\end{tabular}

Table 2: Crystallographic and effective slip tensors as well as the relation between crystallograhic and effictive slip system parameters.

\begin{tabular}{r|ccccccc}
\hline Parameter $\backslash$ mesh no. & 1 & 2 & 3 & 4 & $\mathbf{5}$ & 6 & 7 \\
\hline Elements along diameter & 20 & 30 & 50 & 80 & $\mathbf{1 0 0}$ & 120 & 150 \\
Elements along periphery & 10 & 16 & 30 & 40 & $\mathbf{5 0}$ & 50 & 70 \\
Total no. of elements & 82 & 208 & 622 & 1400 & $\mathbf{2 1 6 2}$ & 2662 & 4602 \\
Init. contact length $a_{c}^{0} / 10^{-3} \mathrm{~mm}$ & 10.58 & 7.653 & 5.325 & 3.228 & $\mathbf{2 . 6 5 9}$ & 2.128 & 1.532 \\
\hline
\end{tabular}

Table 3: Mesh parameters for convergence study. Mesh no. 5 is the mesh used throughout the subsequent analysis. 
Size-effects for wedge indentation in FCC single crystals are modeled A finite strain gradient crystal plasticity theory is developed Numerical results are obtained using a finite element model The supression of GND densities at small indentation depths is quantified A sink-in to pile-up transition is shown to be size dependent for a $90^{\circ}$ indenter 